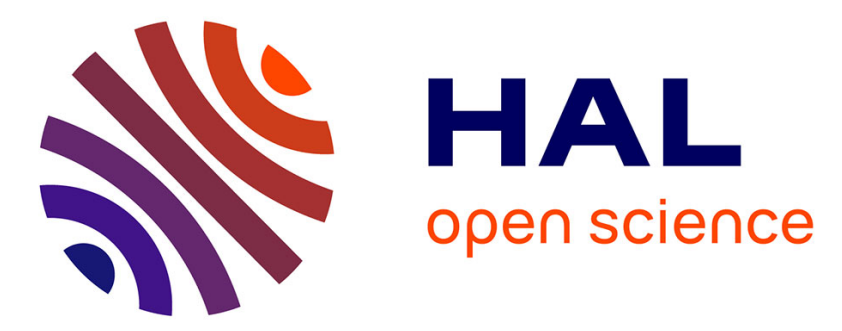

\title{
The Iris Billiard: Critical Geometries for Global Chaos
} Gregory Page, Charles Antoine, Carl P. Dettmann, Julian Talbot

\section{To cite this version:}

Gregory Page, Charles Antoine, Carl P. Dettmann, Julian Talbot. The Iris Billiard: Critical Geometries for Global Chaos. Chaos: An Interdisciplinary Journal of Nonlinear Science, 2020, 30 (12), pp.123105. 10.1063/5.0019968 . hal-03086743

\section{HAL Id: hal-03086743 https://hal.science/hal-03086743}

Submitted on 22 Dec 2020

HAL is a multi-disciplinary open access archive for the deposit and dissemination of scientific research documents, whether they are published or not. The documents may come from teaching and research institutions in France or abroad, or from public or private research centers.
L'archive ouverte pluridisciplinaire HAL, est destinée au dépôt et à la diffusion de documents scientifiques de niveau recherche, publiés ou non, émanant des établissements d'enseignement et de recherche français ou étrangers, des laboratoires publics ou privés. 


\title{
The Iris Billiard:
}

\section{Critical Geometries for Global Chaos.}

\author{
Gregory Page, ${ }^{1, \text { a) }}$ Charles Antoine, ${ }^{1, \text { b) }}$ Carl P. Dettmann, ${ }^{2, c)}$ and Julian Talbot ${ }^{1, d}$ ) \\ 1) Laboratoire de Physique Théorique de la Matière Condensée, Sorbonne Université, Paris, 75005, \\ France. \\ ${ }^{2)}$ School of Mathematics, University of Bristol, Bristol, BS8 1TW, UK.
}

(Dated: 3 November 2020)

We introduce the Iris Billiard, consisting of a point particle enclosed by a unit circle enclosing a central scattering ellipse of fixed elongation (defined as the ratio of the semi-major to the semi-minor axes). When the ellipse degenerates to a circle, the system is integrable, otherwise it displays mixed dynamics. Poincaré sections are displayed for different elongations. Recurrence plots are then applied to the long-term chaotic dynamics of trajectories launched from the unstable period- 2 orbit along the semi-major axis i.e., one that initially alternately collides with the ellipse and the circle. We obtain numerical evidence of a set of critical elongations at which the system transitions to global chaos. The transition is characterized by an endogenous escape event, $\mathscr{E}$, which is the first time a trajectory launched from the unstable period- 2 orbit misses the ellipse. The angle of escape, $\theta_{\text {esc }}$ and distance of closest approach, $d_{\text {min }}$ of the escape event are studied, and are shown to be exquisitely sensitive to the elongation. The survival probability that $\mathscr{E}$ has not occurred after $n$ collisions is shown to follow an exponential distribution.

The Iris Billiard, a unit circle enclosing a central scattering ellipse, of fixed elongation, is a generic system, i.e. one that exhibits mixed dynamics. The chaotic dynamics within this system display incompletely understood features, such as stickiness. Transport barriers within the phase space, which play a fundamental role in the system's mixed dynamics, may be created or destroyed by varying the ellipse elongation. We explore the consequences of such variation on the long-term evolution of chaotic trajectories using recurrence plots. These display features that identify a set of critical elongations that demark a transition to global chaos. The observable properties of the dynamical transition are characterized.

\section{INTRODUCTION}

Billiards $^{1}$ are Hamiltonian systems in which a point particle moves freely within a compact, planar, Euclidean domain. The particle undergoes elastic collisions at the domain's edge and so exhibits dynamics that are purely determined by the interplay between its initial conditions and the confining boundary. These systems exhibit three behaviors: 1) Regular (i.e. periodic or quasi-periodic orbits, as found in circular ${ }^{2,3}$ elliptic ${ }^{4}$ or confocal elliptic ${ }^{5}$ billiards). 2) Ergodic, with orbits that fill the entire phase space, as found in the Sinai ${ }^{6}$, Bunimovich stadium ${ }^{7}$ and cardioid ${ }^{8}$ billiards. 3) Mixed dynamics, i.e. with coexisting regular and irregular trajectories, such as

\footnotetext{
a)Electronic mail: gregorypage823@gmail.com

b)Electronic mail: antoine@lptmc.jussieu.fr

c)Electronic mail: Carl.Dettmann@bristol.ac.uk

d)Electronic mail: talbot@1ptmc.jussieu.fr
}

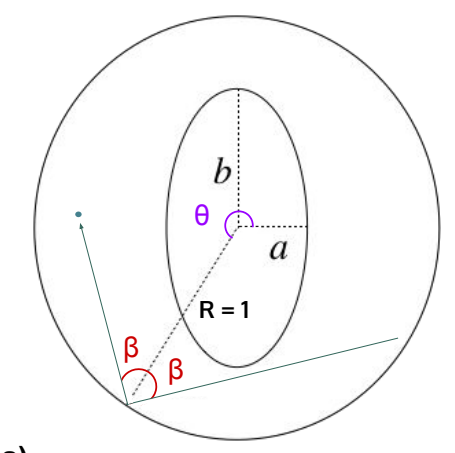

(a)

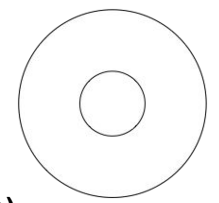

(b)

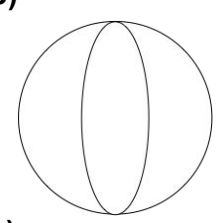

(c)

FIG. 1. The Iris Billiard. (a) Generic configuration: $a \neq b$ (b) Limiting case $a=b$, which forms an annulus. (c) Case $b=1$, which forms two separate crescents $a \in(0,1]$. Orientation angle $\theta$ and reflection angle $\beta$ are shown.

found in the family of limaçon, eccentric annular and mushroom billiards ${ }^{9,10}$.

These systems, which have been the focus of mathematical research ${ }^{2,11-13}$, provide insight into physical phenomena such as encountered in celestial mechanics ${ }^{14,15}$, statistical mechanics ${ }^{6,16,17}$, tokamak physics ${ }^{18,19}$, as well as being important models for quantum chaos ${ }^{20-22}$. Billiard systems connect experimental physics and mathematics through experiments employing both two and three dimensional geometries that may be either open ${ }^{2}$ or closed. Examples include situations where particles or waves are confined to cavities or other homogeneous regions ${ }^{2}$ such as wave guides $^{23}$, electrons in semiconductors confined by electric potentials $^{24}$ and atoms interacting with laser beams ${ }^{25,26}$. Dynamical tunneling between classically isolated phase space regions has also been investigated and observed in both desymmetrized mushroom and eccentric annular su- 
perconducting microwave resonators ${ }^{27,28}$. Escape rates of open billiards is a characteristic that is both experimentally accessible $^{26}$ and important for transport properties of many related systems such as fractal conductance fluctuations ${ }^{29,30}$.

Mixed dynamics have many interesting and unexpected characteristics, such as the existence of dynamical barriers to chaotic transport ${ }^{31}$ and quasi-regular chaotic motion near regions of stability, known as 'stickiness'. ${ }^{32-34}$, of which there are two types ${ }^{35}$. "Internal" stickiness presents in systems with no islands of stability, such as the Bunimovich stadium, or else is due to the presence of marginally unstable periodic orbits (MUPOs) that are completely contained within the chaotic sea, such as in mushroom billiards ${ }^{36}$. "External" stickiness however, arises due to the existence of the boundaries between regular and chaotic regions. Stickiness results in non-exponential decays of both the time-correlation functions and Poincaré recurrence distributions of the system's chaotic dynamics $^{37-40}$.

To understand these behaviors, recurrences ${ }^{41,42}$, which are events characterized by a given trajectory occupying a state close to one already visited, are often considered. Recurrence plots (RPs) ${ }^{43,44}$ permit the quantitative and qualitative study of this property. This tool has already been applied in the fields of economy ${ }^{45}$, physiology ${ }^{46}$, ecology $^{47}$, neuroscience ${ }^{48}$ and astrophysics ${ }^{49}$. Although recurrence statistics have been extensively studied in billiards ${ }^{31,34,39}$, RPs appear to have only been applied to position recurrences in a two particle billiard system ${ }^{50}$ and, more recently, to the Poincaré section of an eccentric annular billiard ${ }^{51}$. In this paper, time recurrences in the Poincaré section will be considered.

Section II fully describes the model. Section III presents a set of Poincaré sections for different ellipses, and details their features. Section IV applies RPs and a new time measure to chaotic trajectories starting with the unstable period- 2 orbit for many ellipse parameters. Section V identifies and further studies the endogenous escape event. Section VI discusses the results and concludes.

\section{THE SYSTEM}

The billiard domain, $B \subset R^{2}$, has a two-part, continuous, boundary, $\partial B=\bigcup^{i} \partial B_{i}$, where each $\partial B_{i}$ is piece-wise smooth. The initial conditions of a given trajectory are defined by the arc length distance, $s=\theta \in(-\pi, \pi]$, along the outer circular boundary, and the initial direction of motion, described by the angle between the initial velocity and the center-facing normal to the outer boundary, $\beta \in(-\pi / 2, \pi / 2]$ : See Fig. 1 . At any instant the point particle, mass $m=1$, is described by its position, $q \in B$, and momentum, $|p|=1$. The dynamics is governed by the Hamiltonian:
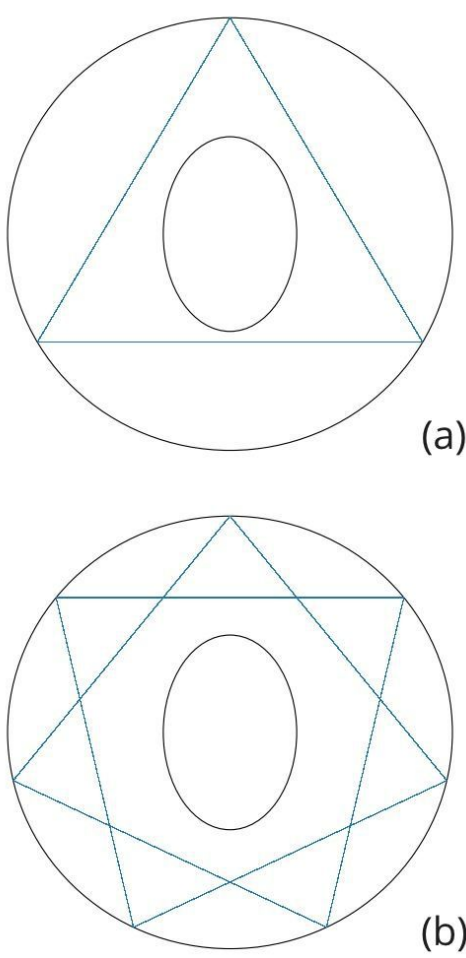

(b)

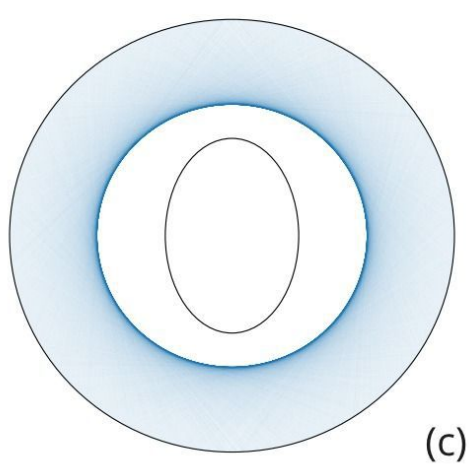

FIG. 2. A selection of regular, circle map, trajectories. a) A periodic orbit with frequency ratio $\omega_{1} / \omega_{2}=1 / 3$. b) A periodic orbit with frequency ratio $\omega_{1} / \omega_{2}=2 / 7$. c) A quasi-periodic orbit with frequency ratio $\omega_{1} / \omega_{2}=1 / \sqrt{2}$ that would densely fill the annulus if allowed to run for infinite time. All trajectories were generated with $10^{4}$ collisions.

$$
H(q, p)= \begin{cases}p^{2} / 2, & q \in B / \partial B . \\ \infty, & q \in \partial B .\end{cases}
$$

The infinite boundary potential causes every collision to be elastic. Therefore, the component of the momentum projected onto the normal at the point of each collision changes sign, while the tangential component stays constant. The momentum vector, $\mathbf{p}_{\mathbf{i}+\mathbf{1}}$ after the $i^{t h}$ collision at point $q_{i}$ is:

$$
\mathbf{p}_{\mathbf{i}+\mathbf{1}}=\mathbf{p}_{\mathbf{i}}-2\left[\mathbf{p}_{\mathbf{i}} \cdot \hat{\mathbf{n}}\left(\mathbf{q}_{\mathbf{i}}\right)\right] \hat{\mathbf{n}}\left(\mathbf{q}_{\mathbf{i}}\right) .
$$



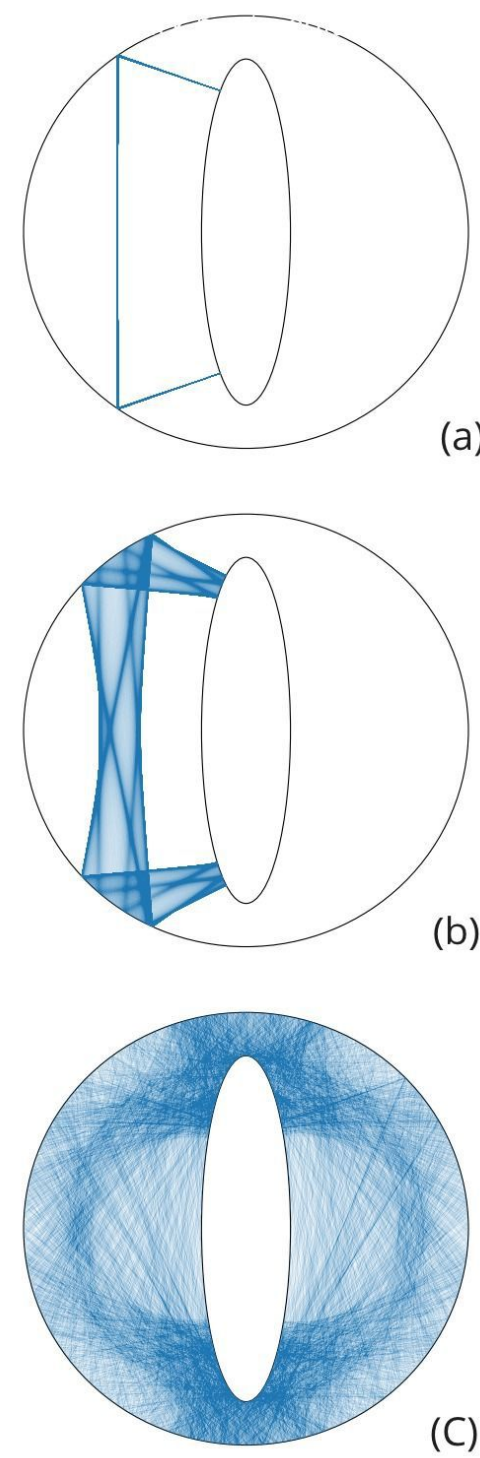

(C)

FIG. 3. A selection of orbits that collide with the ellipse. Geometry: $a=0.2, e=4$. a) Periodic orbit: initial conditions: $\theta=2.19, \beta=$ $0.577 \mathrm{~b})$ Quasi-periodic orbit: initial conditions: $\theta=2.48, \beta=$ 0.577. c) Chaotic orbit: initial conditions: $\theta=3, \beta=0.577$. All trajectories were generated with $10^{4}$ collisions.

$\hat{\mathbf{n}}\left(\mathbf{q}_{\mathbf{i}}\right)$ is the normal of the boundary at each collision. The equation of the ellipse is:

$$
\frac{x^{2}}{a^{2}}+\frac{y^{2}}{b^{2}}=1
$$

Where $a$ and $b$ are the semi-major, and minor axes, $(b \in(0,1]$ and $a \in(0, b])$. We use the elongation, $e=b / a$ as a measure of the breaking of the system's symmetry. When $e=1$, the system reduces to the centered annulus, which is totally symmetric, and therefore integrable by conservation of angular momentum.
All possible trajectories fall into one of two mutually exclusive categories: those that never hit the central ellipse and those that do. The first type, illustrated by Fig. 2, are characterized for all quasiperiodic orbits by an angle $\beta$ that obeys the following tangency condition:

$$
\beta \geq \beta_{c}=\arcsin b,
$$

$b$ is the radius of the inaccessible circular region, defined by a caustic edge, as seen in Fig $2 c$. The trajectories are regular (i.e. either periodic or quasi-periodic, with constant $\beta$ ), and are given by the unperturbed circle map:

$$
\theta_{n+1}=\theta_{n}+\Omega \bmod 2 \pi, \text { with } \Omega=\pi-2 \beta .
$$

If $\Omega / 2 \pi$ is rational, the motion may be represented as a quotient of coprime numbers such that: $\Omega=2 \pi \omega_{1} / \omega_{2}$ with $\omega_{1}, \omega_{2} \in \mathbb{Z}^{+}$. In this case, the orbit closes on itself after a finite number of iterations, i.e. it is periodic, and will form polygons as in Fig 2 a and b. If $\Omega / 2 \pi$ is irrational, the sequence $\left\{\theta_{n}\right\}$ ergodically fills $[0,2 \pi]$, as time tends to infinity, as shown in Fig 2 c. It is well known that rational approximations of some irrational frequency, are obtained through its continued fraction representation ${ }^{52}$.

Numbers can be irrational to different degrees, the highest of which is the golden mean, represented by a continued fraction whose partial quotients are all equal to 1 , and is an element of the set of noble frequencies, whose partial quotients always end in ones, although the first quotients may be different. The transport barriers defined by these frequencies are more robust under perturbation, as has been verified in work that applied Greene's residue criterion ${ }^{53}$, to numerically determine the frequency of the final destroyed invariant transport barrier for both the standard map and the double pendulum ${ }^{54}$.

If the tangency condition is not satisfied, some rational $\Omega=$ $\omega_{1} / \omega_{2}$ orbits may continue to exist over a finite range of ellipse elongations. These trajectories form $\omega_{2}$-polygons whose radius depends of intersection of the enclosed caustic is:

$$
r_{\omega_{1}, \omega_{2}}=\left|\cos \left(\frac{\omega_{1} \pi}{\omega_{2}}\right)\right|
$$

The minimum value of $e$ at which the rational polygon may exist at some orientation without intersecting the ellipse is :

$e_{\text {min }}=\left[\frac{1}{\cos ^{2}\left(\pi / \kappa(n) \omega_{2}\right)}\left(\frac{r_{\omega_{1}, \omega_{2}}^{2}}{a^{2}}-1\right)+1\right]^{1 / 2} \omega_{2} \in\{3,4,5 \ldots\}$

where:

$$
\kappa\left(\omega_{2}\right)=1+\omega_{2} \bmod 2 .
$$

See Appendix A for the derivation. Fixing $\omega_{1}$ and taking the limit $\omega_{2} \rightarrow \infty$ returns the limit: 

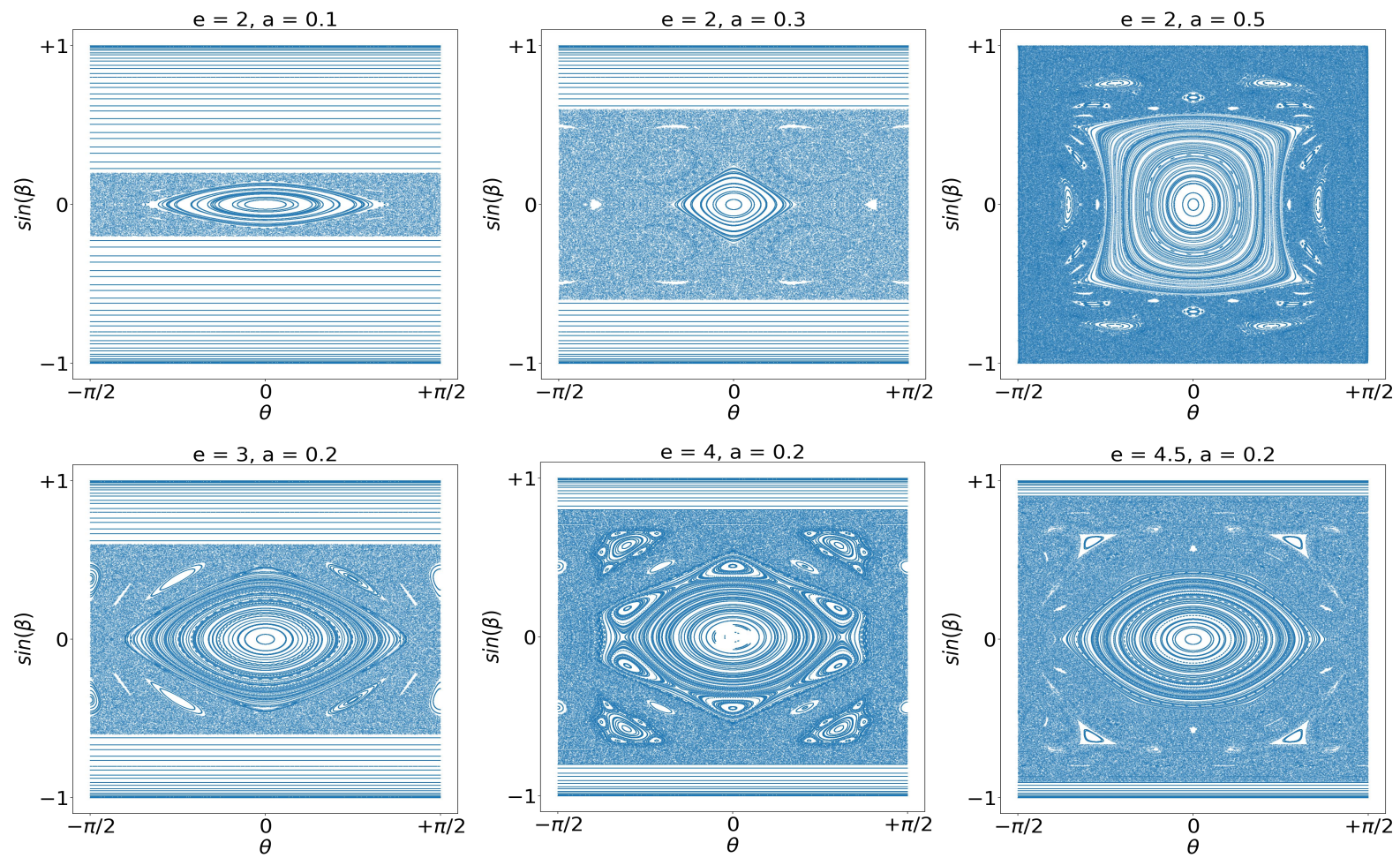

FIG. 4. A set of complete Poincaré sections. Top row: Constant elongation: $e=2$, for increasing semi-minor axis from left to right. Bottom row: Constant semi-minor axis: $a=0.2$, for increasing elongation from left to right.

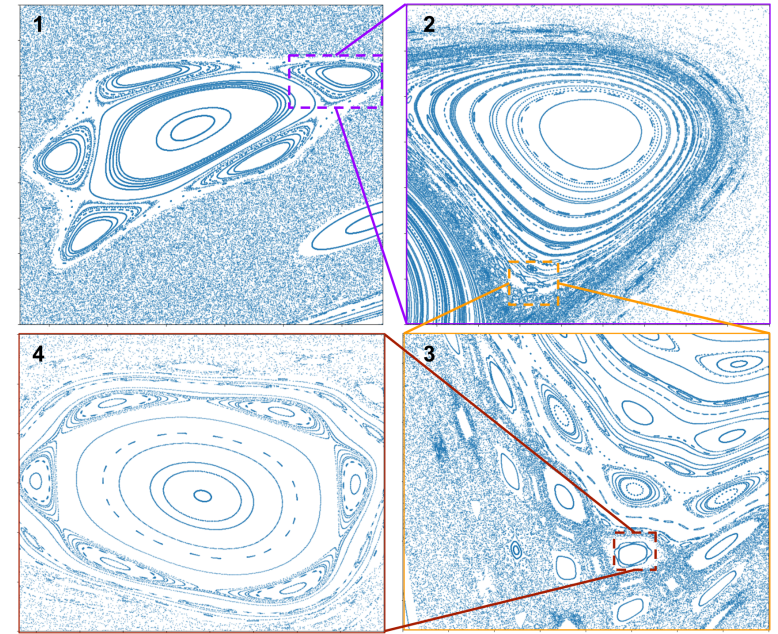

FIG. 5. 1-4, Successive magnifications of the archipelago seen in the top left of the Poincaré section obtained for $e=4, a=0.2$. (Figure 4, bottom, centre).

$$
\lim _{\omega_{2} \rightarrow \infty} e_{\min }=1 / a
$$

On the contrary, the maximum value of $e$, at which the $\omega_{2}$ polygon intersects the ellipse for any orientation is:

$$
e_{\max }=r_{\omega_{1}, \omega_{2}} / a
$$

and so approaches the same limit as $e_{\min }$ when $\omega_{2} \rightarrow \infty$.

The second type of trajectory, that involves collisions with the ellipse, illustrated by Fig. 3, results in rotational and librational periodic and quasi-periodic orbits (i.e. $\beta \neq$ constant) as well as chaotic orbits. Appendix B includes a stability analysis for the simplest period- 2 orbits along the axes of symmetry of the ellipse. The orbit along the semi-minor axis is stable for all elongations. In contrast, the orbit along the semi-major axis is unstable for all elongations greater than one, where all orbits are stable. However, the range of dynamics in Fig. 3 can only be completely visualized by considering the phase space.

\section{POINCARÉ SECTIONS}

The phase space is a set of points that fully describe the system. For planar billiard systems, it is four dimensional, $\left(x, y, v_{x}, v_{y}\right)$. However, the complete description of an orbit can be shown using a two-dimensional Poincaré section, via Birkhoff coordinates $^{55},(\theta, \sin (\beta))$.

$\theta$ is the arclength of the outer circle collision. Due to the system's axial symmetry, $\theta$ can be restricted to the range $\theta \in(-\pi / 2, \pi / 2] . \quad \sin \beta \in(-1,1)$ is the momentum component, at the point of collision, tangential to the boundary. 
The periodicity in $\theta$ makes the Poincare section topologically equivalent to an annulus, $\mathbb{S} \times[-1,1]$.

Fig. 4 shows a series of sections constructed by trajectories launched from initial conditions spanning $\theta, \beta \in$ $(-\pi / 2, \pi / 2)$. If the tangency condition, Eq.(4), is obeyed, trajectories follow the rotational curves above and below the central mixed region, defined by $\beta=$ constant. Lines corresponding to periodic, but from a disjoint set of initial conditions, and rotational quasi-periodic motion shown in Fig. 2, smoothly wind around the annulus. These circles are homotopically non-trivial and are known as 'rotational circles'. Increasing the elongation of the ellipse, $e$, until the tangency condition, Eq.(4), is just violated, causes the trajectory to make contact with the ellipse, therefore deforming the orbit's associated invariant rotational circle in the Poincaré section. The curves often persist with increasing elongation, as predicted by KAM theory, and there is, consequently, no flux of trajectories between the regions partitioned by the deformed circle. As the elongation is further increased, however, the invariant curves will be increasingly deformed until some critical value is reached, (each corresponding to the quasi-periodic frequency ratio of the deformed curve in question), at which point the curve is destroyed. When $\beta<\beta_{c}$, the Poincaré section displays mixed dynamics. i.e. it is divided into several invariant components.

The center of every Poincaré section presented, i.e. $\theta=0, \beta=0$, or equivalently $\pm \pi$, is an elliptical fixed point for all geometries, corresponding to the stable period- 2 orbit along the semi-minor axis. Sets of concentric, homotopically trivial circles, representing librational quasi-periodic motion (as seen, for example, in Fig. 3b) surround the stable period-2 orbit, for all geometries, as well as from other geometrydependent elliptical periodic orbits. It is thought that these curves only cause a limited impediment to the diffusion of chaotic orbits since they do not encircle the entire annulus ${ }^{53}$. Librational circles occur in concentric sets. The outermost forms the critical boundary of the island of stability, which can be destroyed by an arbitrarily small perturbation.

The hyperbolic period- 2 orbit along the ellipse semi-major axis is always within the chaotic sea. (The exception to this is when $e=1$ i.e. when the system is integrable). Boundaries between the regular and chaotic components of the phase space are often characterized by scale invariant structures. Such features are illustrated by Fig. 5, in which an island archipelago is magnified indicating where a critical curve used to lie. Insets 2-4 clearly show scale invariant structures that reveal the different dynamics present inside the islands, i.e. quasi-periodic trajectories enclosing narrow stochastic layers. These island chains create partial barriers to chaotic transport, and are the source of external stickiness.

\section{RECURRENCE PLOTS AND QUANTIFICATION ANALYSIS.}

The Poincaré recurrence theorem states that for a finite measure preserving transformation, almost every point in a finite measure set will return to its neighborhood infinitely many times. Therefore, even though very close chaotic trajectories exponentially deviate in finite time ${ }^{56}$, they must, eventually, return arbitrarily close to their initial conditions and evolve in ways similar to before ${ }^{57}$. Although this theorem gives no indication of the frequency at which recurrences occur, RPs allow the quantitative and qualitative study of this feature ${ }^{44}$. During an orbit of $N_{c o l}$ total collisions, there are $N_{\circ}$ collisions with the outer boundary. The time evolution of the points corresponding to a trajectory in the Poincaré section can be labeled as : $\left\{\boldsymbol{v}_{i}\right\} \quad i=1 \ldots N_{\circ}$. A state, $\boldsymbol{v}_{\boldsymbol{j}}$, is defined as recurrent to a former state, $\boldsymbol{v}_{\boldsymbol{i}}$, if sufficiently close.

The $L_{\infty}$ norm is used to define the neighborhood around each point, which defines a square of length $\varepsilon$ with $\boldsymbol{v}_{\boldsymbol{i}}$ at its center, such that $\boldsymbol{v}_{\boldsymbol{j}}$ is a recurrent state of $\boldsymbol{v}_{\boldsymbol{i}}$ if and only if the two states lie within the same square region. The value of $\varepsilon$ is important. If too small, no recurrences would be recorded in a finite time. If too big, every point would be recorded as a recurrence of every other point, leading to artifacts unrelated to the dynamics. Although these artifacts may be analytically determined for simple periodic and quasi-periodic motions ${ }^{58}$, in general they cannot be completely removed by any known means. We follow the usual convention of defining $\varepsilon$ as $10 \%$ of the width of its corresponding phase component ${ }^{43}$.

The binary, $N_{\circ} \times N_{\circ}$, recurrence matrix is defined as:

$$
\mathbf{R}_{i, j}=\Theta\left(\varepsilon-\left\|\boldsymbol{v}_{\boldsymbol{i}}-\boldsymbol{v}_{\boldsymbol{j}}\right\|\right), \quad i, j=1 \ldots N_{\circ},
$$

where $\Theta(\cdot)$ is the Heaviside function.

RPs are the graphical representation of $\mathbf{R}_{i, j}$. The value '1', encoded by a black point, indicates that $\left|\boldsymbol{v}_{\boldsymbol{i}}-\boldsymbol{v}_{\boldsymbol{j}}\right|<\boldsymbol{\varepsilon}$. Otherwise, points are blank, representing the value ' 0 '. All RPs will show a diagonal line, i.e. $\mathbf{R}_{i, j}=1 \forall i=j$, known as the Line of Identity (LOI). RPs display many patterns associated with different behaviors. At the small scale, they exhibit isolated points, diagonal lines and vertical lines; (the combination of the latter two results in rectangular clusters of recurrence points $)^{43}$. Single, isolated recurrence points indicate that a state is rare, or only briefly persists. Diagonal lines, running parallel to the LOI, of length $l$ occur when part of a trajectory runs almost in same phase neighborhood as a previous portion, for $l$ segments. Finally, vertical lines indicate time intervals in which a state is either stationary or changes very slowly. Applying RPs to the rotational periodic and quasi-periodic dynamics gives expected results, such as required by the Steinhaus conjecture (three gap theorem $)^{59}$. Reference ${ }^{51}$ provides an overview of these behaviors in the context of the eccentric annular billiard. 

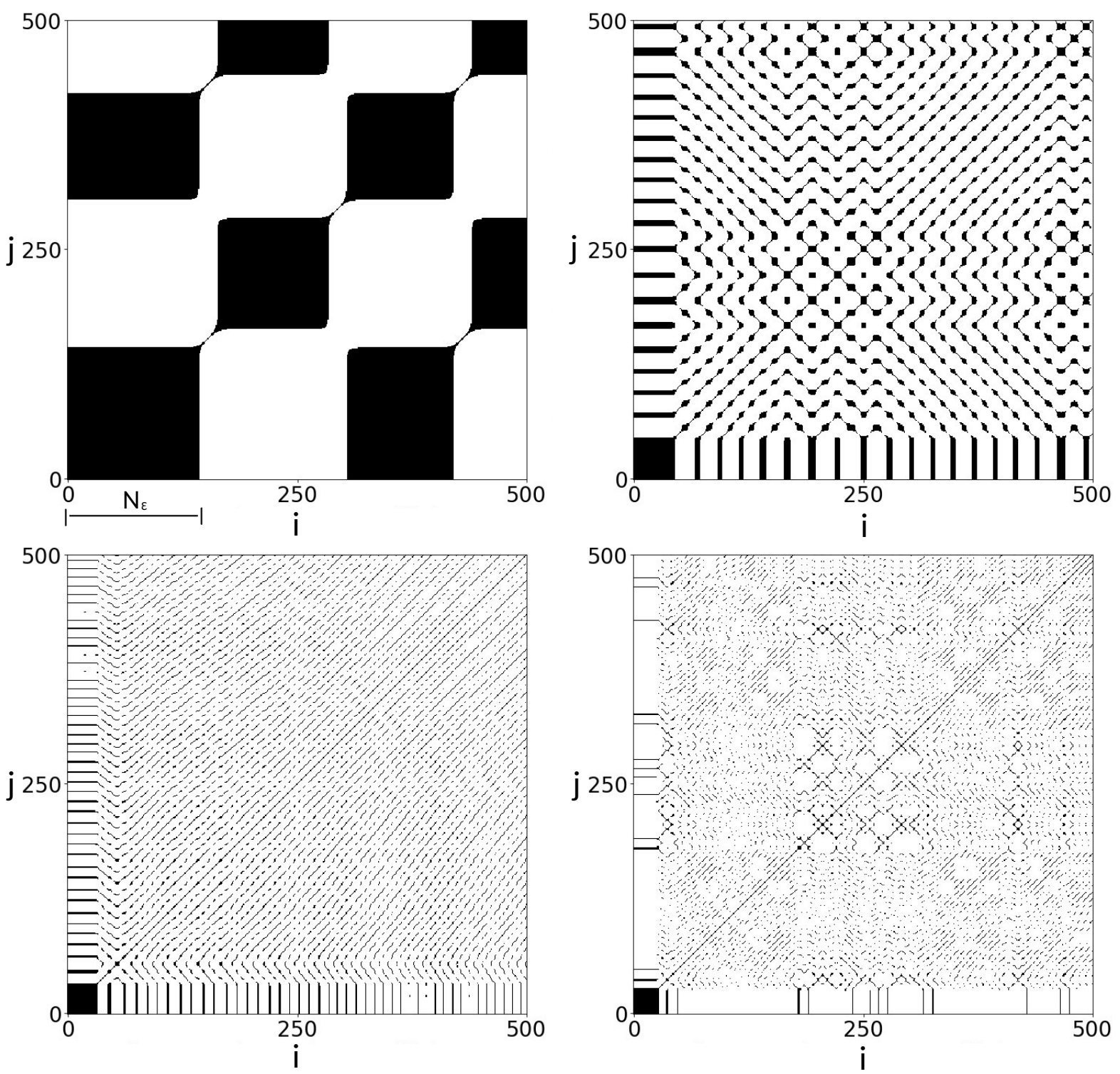

FIG. 6. Recurrence Plots (RPs), visualizing the dynamics resulting from the unstable period- 2 orbit for different elongations. $a=0.2$ Top left: $e=1.01$, where the measure of interest; $N_{\varepsilon}$ is indicated, Top right: $e=1.1$, Bottom left: $e=1.2$, Bottom right: $e=1.3$, for which dynamical transitions, as indicated by the variation of density of recurrence points, are clearly present.

To explore the relationship between chaotic dynamics and geometry, we studied the recurrence properties of the longterm motion launched as close to the unstable period-2 orbit as numerically possible. RPs of a $10^{3}$ iteration trajectory, for $a=0.2, e=1.01,1.1,1.2,1.3$ are illustrated in Fig.6.

Although many tools already exist to quantify most features in the RPs presented, we carry out a simple analysis by introducing a new time measure, $N_{\varepsilon}$ : The number of collisions with the outer boundary before the particle exits its initial $L_{\infty}, \varepsilon$-neighborhood for the first time. This manifests as the black box in the bottom left-hand corner of all four plots in Fig.6.
Fig.7 shows the evolution of $N_{\varepsilon} / N_{\circ}$, for a fixed value of $N_{c o l}=10^{4}$, for different ellipse parameters. For all values of $a$ considered, as $e \rightarrow 1$, so does $N_{\varepsilon} / N_{\circ}$. This is because the initial period-2 orbit approaches stability, as demonstrated in Appendix B, so it always remains in its initial neighborhood. Similarly, as $e \rightarrow 1 / a, N_{\varepsilon} / N_{\circ}$ again approaches unity as the period-2 orbits approaches stability. This can be expected as, intuitively, as the region becomes more confined, the distance the particle traverses between each collision approaches zero, meaning that small deviations from the initial conditions will have an increasingly negligible effect on the orbits stability matrix (see Appendix B, section A2 ).

The most important feature of the main plot in Fig. 7 is the 


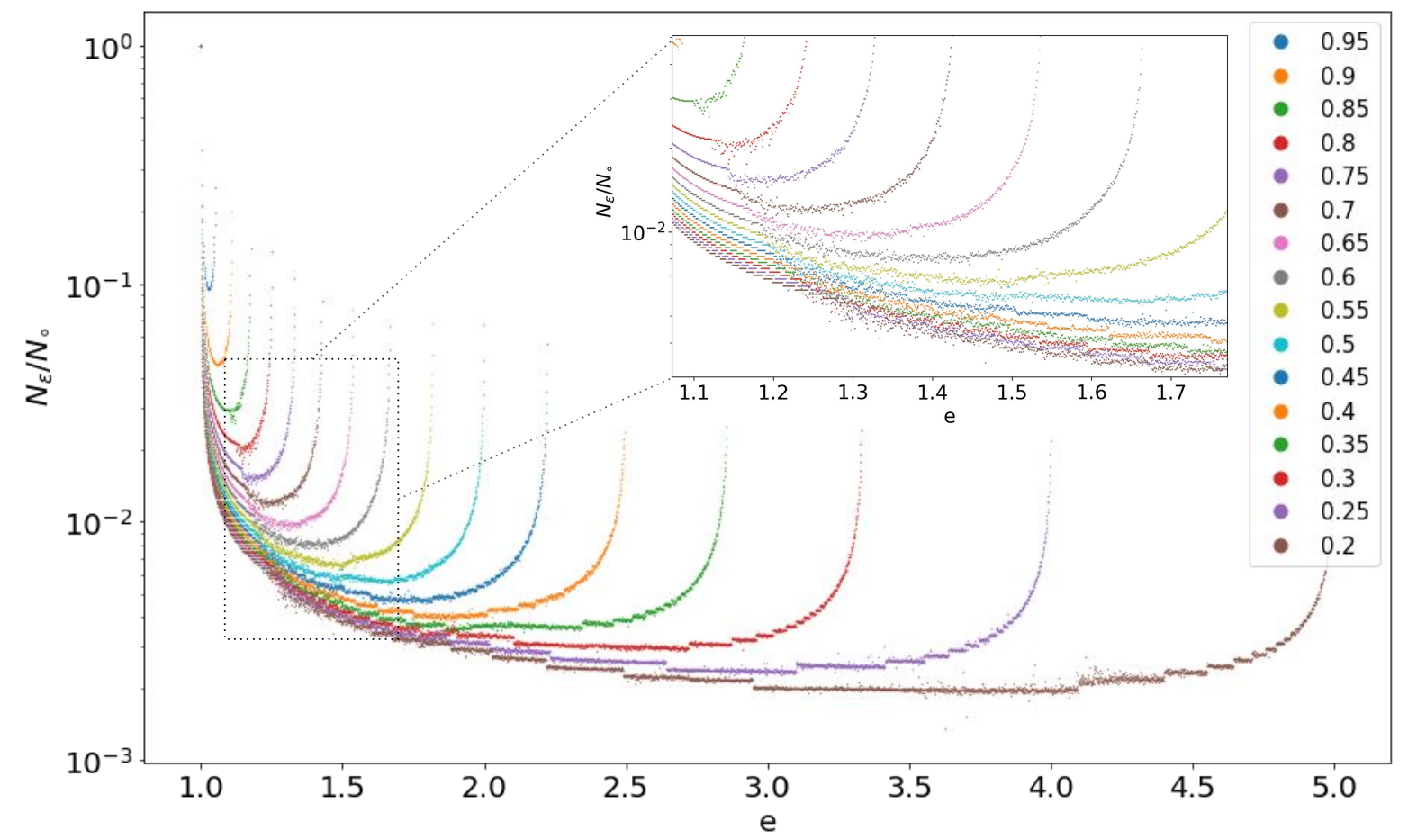

FIG. 7. The ratio of the number of collisions within the initial neighbourhood of the unstable period-2 orbit, $N_{\varepsilon}$, to the total number of collisions with the outer circular boundary, $N_{\circ}$, as a function of the elongation, $e$. A logarithmic scale is used to show the transition point clearly on each curve. $X$-axis: The elongation of the inner scatterer for different values of a, each identified by a color in the legend. In each case, total trajectory length, $N_{c o l}=10^{4}$.

transition of $N_{\varepsilon} / N_{\circ}$ from smooth to rough. This happens at different values of elongation for each value of semi-minor axis, $a$, and is insensitive to changes in numerical precision. To understand this phenomenon, recall that the trajectory begins as the unstable period- 2 orbit, i.e. the particle collides with the boundary at every other iteration. When $e=1$, $N_{\varepsilon}=N_{\circ}=N_{\text {col }} / 2$. As $e$ increases, $N_{\varepsilon} / N_{\circ}$ smoothly evolves until a critical value of the elongation, $e=e_{\text {crit }}(a)$. This critical geometry causes a non-zero probability that the particle, having just collided with the circular outer boundary, will miss the ellipse, and collide again with the outer circle, therefore breaking the parity condition. The first time this event occurs in a trajectory will be referred to as the endogenous escape event, or $\mathscr{E}$ for brevity. This must not be confused with the escape events normally studied in open billiard systems, as the hole through which the particle is escaping in this case is intrinsic to the system's phase space. The true value of $e_{c r i t}$ depends neither on $\varepsilon$, nor the numerical precision chosen. $e_{c r i t}(a)$ is defined as the elongation for which the following is true:

$$
\lim _{N_{c o l} \rightarrow \infty}\left(N_{\circ}-\frac{N_{c o l}}{2}\right)=1 .
$$

To estimate $e_{\text {crit }}$ for a given value of a, we used the following algorithm. The initial value of $e$ is set to $1+\delta e$ with $\delta e=0.02$ in the results presented, and a trajectory of $2 \times 10^{6}$ collisions is launched near the unstable period 2 orbit. If $N_{\circ}=N_{\text {col }} / 2$, the current value of $e$ is increased by $\delta e$ and another trajectory is launched. This is continued until $N_{\circ}>N_{c o l} / 2$ and $e_{c r i t}$ is taken as the current value of $e$.

Due to finite simulation times, any obtained value of $e_{\text {crit }}$ will inevitably be an overestimation. Figure. 8 shows that $e_{\text {crit }}(a)$ demarks the elongations that permit two classes of behavior by reconstructing, and including as insets, the long-term trajectories of the unstable period-2 orbit for values below and above $e_{\text {crit }}(a)$. When $e<e_{\text {crit }}(a)$, the trajectory explores an extended stochastic region that is bounded by a rotational KAM curve/surface. This rotational curve is destroyed at $e=e_{\text {crit }}(a)$, i.e. holes are created which allow the trajectory to escape its reduced chaotic portion of the phase space after a finite number of iterations. By simple inspection of Fig.8, no other similar transitions are observed for elongations beyond the critical value.

For values of elongation below the critical value, $e_{\text {crit }}(a)$, the trajectories exploring the bounded chaotic region are clearly still subject to both position and momentum diffusion, yet under the constraint that the point particle will always alternately collide with the circle and the ellipse. In this case, one may symbolize the trajectory, $\mathbf{T}$ as:

$$
\boldsymbol{T}=[\ldots, e, c, e, c, e, c, e, c \ldots]
$$




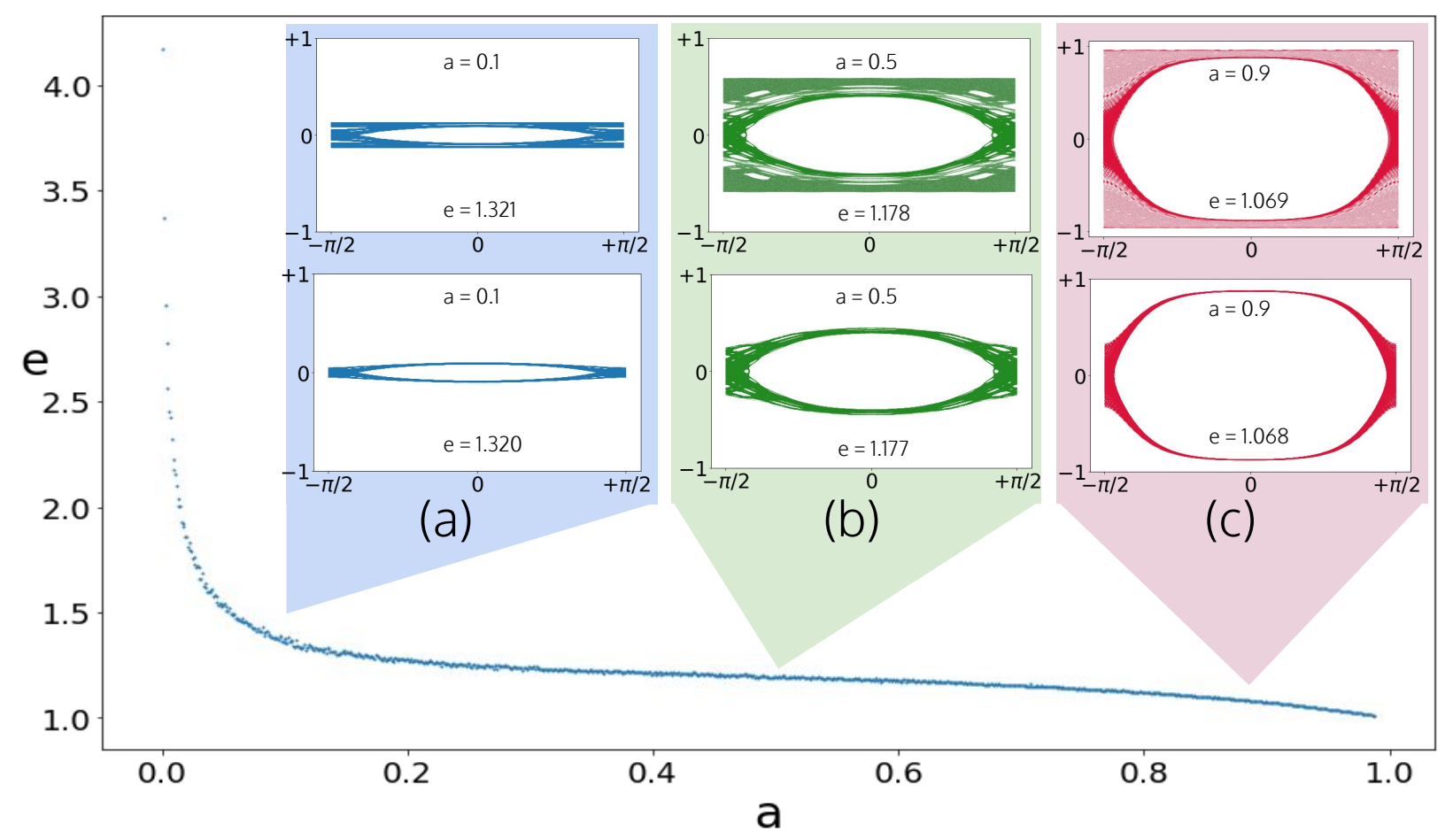

FIG. 8. Evolution of $e_{\text {crit }}(a)$ for $a \in(0,1)$. Trajectories of length $N_{c o l}=2 \times 10^{6}$ were used to obtain each point. The curve indicates the elongation at which the final rotational curve is destroyed as a function of $a$. The subfigures show the Poincare section occupation of the long time chaotic trajectory $\left(N_{c o l}=10^{7}\right)$ for elongations just above and below $e_{\text {crit }}$ (a) $a=0.1$, below: $e=1.320$ above: $e=1.321$. (b) $a=0.5$, below: $e=1.178$ above: $e=1.179$. (c) $a=0.9$, below: $e=1.068$ above: $e=1.069$.

where $c$ and $e$ denote collisions with the circle and ellipse, respectively. For $e>e_{\text {crit }}$, this constraint no longer applies. The moment this parity condition is broken implies the dynamical transition has occurred, and is characterized by $\mathscr{E}$, i.e. the first passage of the particle's trajectory from one major fractal subset to the rest of the chaotic phase space. In this case, the trajectory may now be symbolized as:

$$
\boldsymbol{T}=[\ldots, c_{n_{e s c}-4}, e_{n_{e s c}-3}, c_{n_{e s c}-2}, e_{n_{e s c}-1}, \overbrace{c_{e s c}}^{\mathscr{E}} \mathbf{c}, \ldots] .
$$

The first, bold, consecutive c represents $\mathscr{E}$ by hitting the outer circular boundary twice in a row, which, as before specified, never occurs when $e<e_{c r i t}(a)$. We note that $\lim _{e \rightarrow e_{c r i t}} n_{e s c}=\infty$. Without explicit numerical calculation, it seems impossible to know the symbolic order following $\mathscr{E}$. The set $\left[\mathscr{E}_{1}, \mathscr{E}_{2}, \mathscr{E}_{3} \ldots\right]$, is defined as the first, second, third, etc. times the trajectory consecutively hits the outer boundary after $\mathscr{E}$.

\section{STUDY OF $\mathscr{E}$}

To characterize the endogenous escape event, we introduce the angle, $\theta_{e s c}$, at which the escaping segment comes closest to the ellipse and the distance of closest approach, $d_{\min }$, shown in Fig. 9, are presented in Figs.10 - 20. These quantities were obtained by noting that, at the point of closest approach, the trajectory lies parallel to the ellipse at the closest point on the ellipse circumference. Appendix D provides the geometrical derivation of these quantities and further details.

\section{A. Numerical procedure}

To ensure that the results presented reflect the global properties of the chaotic region of the phase space, the $N_{\text {sample }}=10^{6}$ trajectories studied were launched from slightly different initial conditions, within the neighborhood of the unstable period 2 orbit. The starting position on the outer boundary is defined by:

$$
\theta_{k}=(-1)^{k} \pi / 2+\varepsilon_{\theta} \eta_{\theta}
$$

Where $1 \leq k \leq N_{\text {sample. }}$. The starting orientation is given by:

$$
\beta_{k}=\varepsilon_{\beta} \eta_{\beta}
$$

where $\varepsilon_{\beta}=\varepsilon_{\theta}=10^{-10} . \eta_{\theta}, \eta_{\beta}$ are random numbers chosen from independent uniform random distributions between -1 
and +1 , i.e. the starting position of the two unstable orbits are given very slight, unbiased variations, which will cause the trajectories to exponentially deviate over a finite time and, therefore, follow very different trajectories within the reduced chaotic phase space before escaping. The following results displayed only minor variations upon setting $\varepsilon_{\theta}$ or $\varepsilon_{\beta}$ to zero.

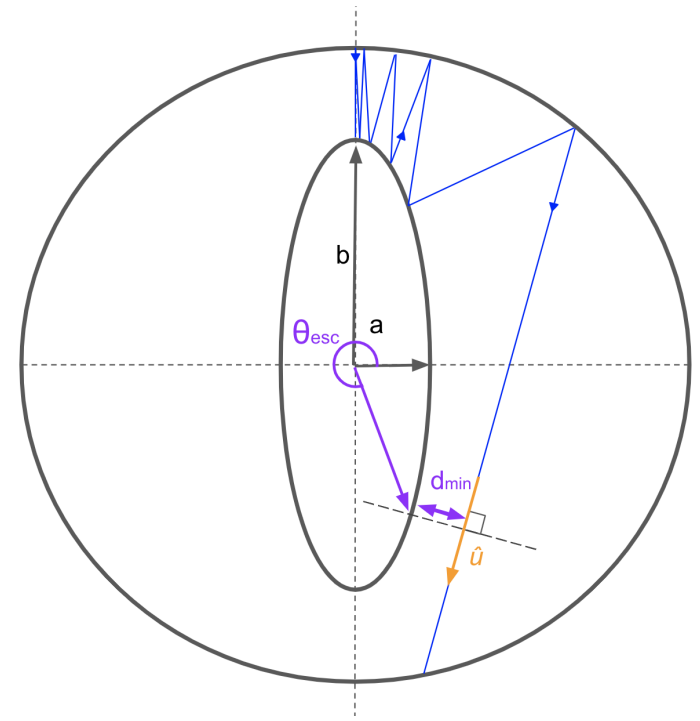

FIG. 9. An illustration of the endogenous dynamical escape event, $\mathscr{E}$, of a trajectory launched from the unstable period 2 orbit, that signals the transition to global chaos. The relevant observable variables, $\theta_{\text {esc }}$ and $d_{\min }$ are indicated. The unit vector of the escape segment, $\hat{u}$ is also shown.

\section{B. Results}

Heatmaps showing the distributions of $\theta_{\text {esc }}$ and $d_{\min }$ for $\mathscr{E}, \mathscr{E} 10$ and $\mathscr{E}_{50}$ are presented in Figs. 10, 12, 14, 16, 18 and 20. Results for $a=0.1,0.5,0.9$ are collected for two values of $e$ above and approaching $e_{\text {crit }}$. Survival probabilities before $\mathscr{E}$ are presented in Figs. 11, 13, 15, 17 and 21. These figures also show a semi-log linear fit, $\log \left(P_{\text {survival }}\right)=A n+B$, over the domain demarked by the two vertical red dashed lines, using the method of least squares. Both $A$ and $B$ are given within the figures. $n_{\min }$ is the minimum recorded value before any escape, and so may be thought of as a deterministic dead time. No power-law behaviors were observed. The heatmaps were constructed by discretizing the $\theta_{e s c}$ and $d_{\min }$ values for each geometry studied, and plotting the correspondences between the two values on a $2 \mathrm{D}$ histogram. The global heatmaps were made with a resolution of $L \times L$ cells where $L=500$. A colorbar is used to count the number of events recorded per 'cell'. Units given as 'counts per bin': $c p b$. The following results are symmetric around $\theta_{e s c}=0, \pi$ and $\theta_{e s c}=-\pi / 2, \pi / 2$.

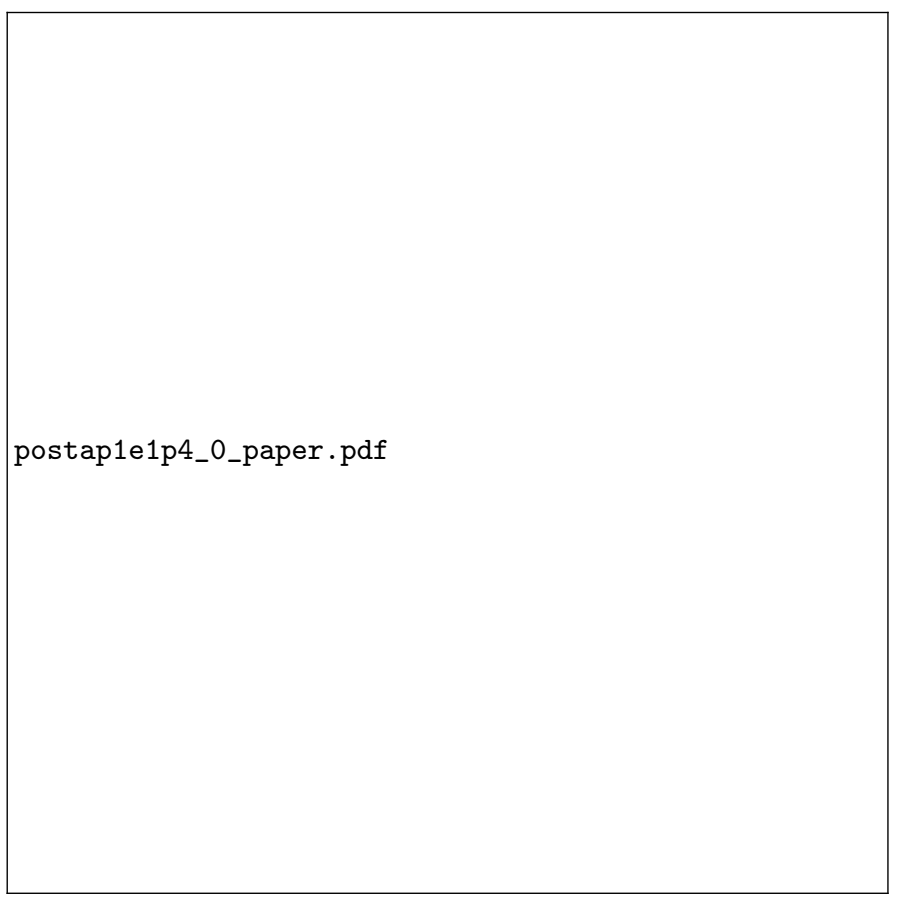

(a) $\mathscr{E}$

postap1e1p4_10_paper.pdf

(b) $\mathscr{E}_{10}$

postap1e1p4_50_paper.pdf 


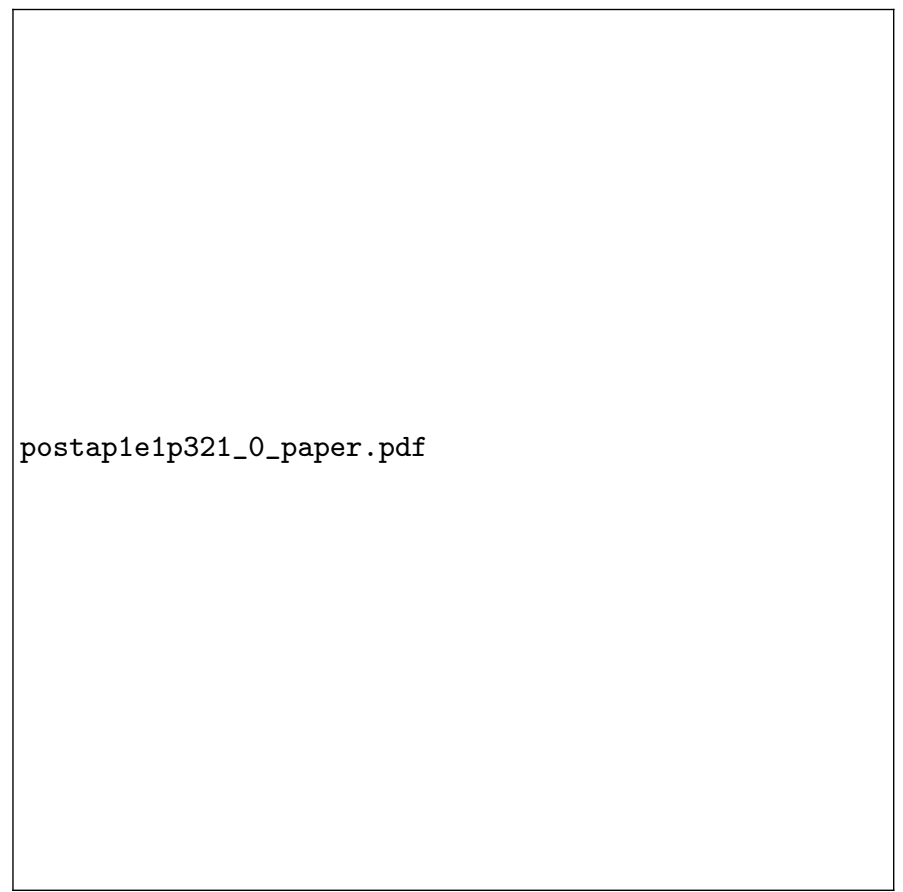

(a) $\mathscr{E}$

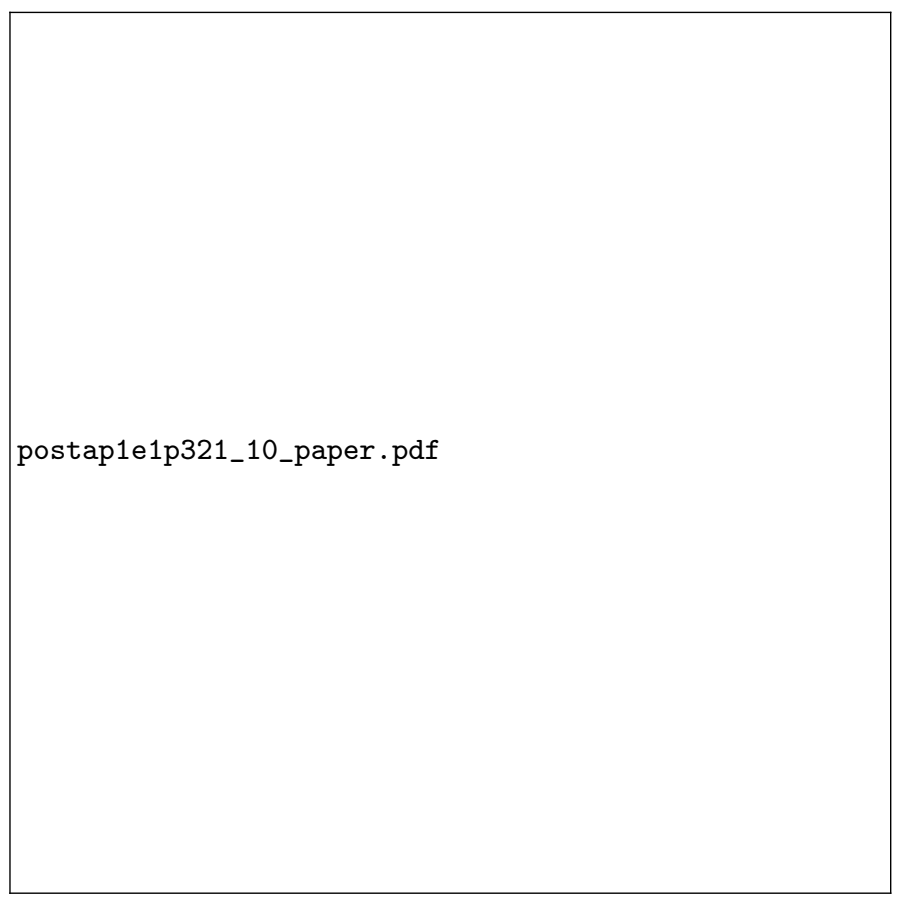

(b) $\mathscr{E}_{10}$

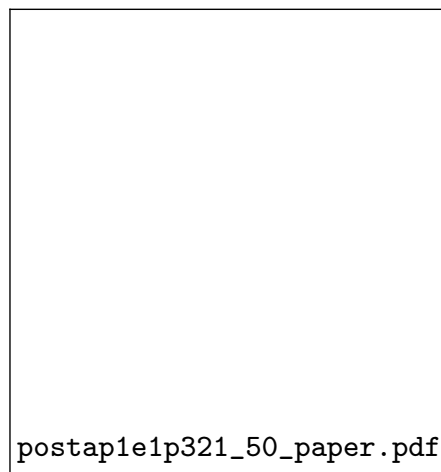

postap1e1p321_50_paper.pdf

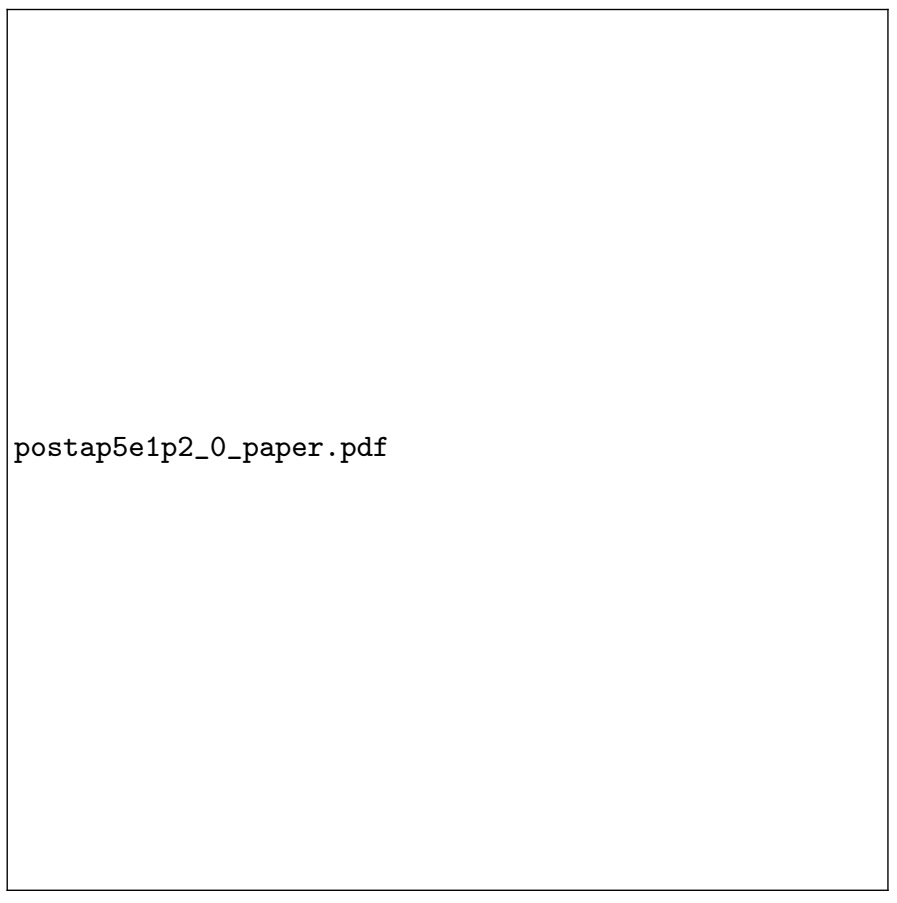

(a) $\mathscr{E}$

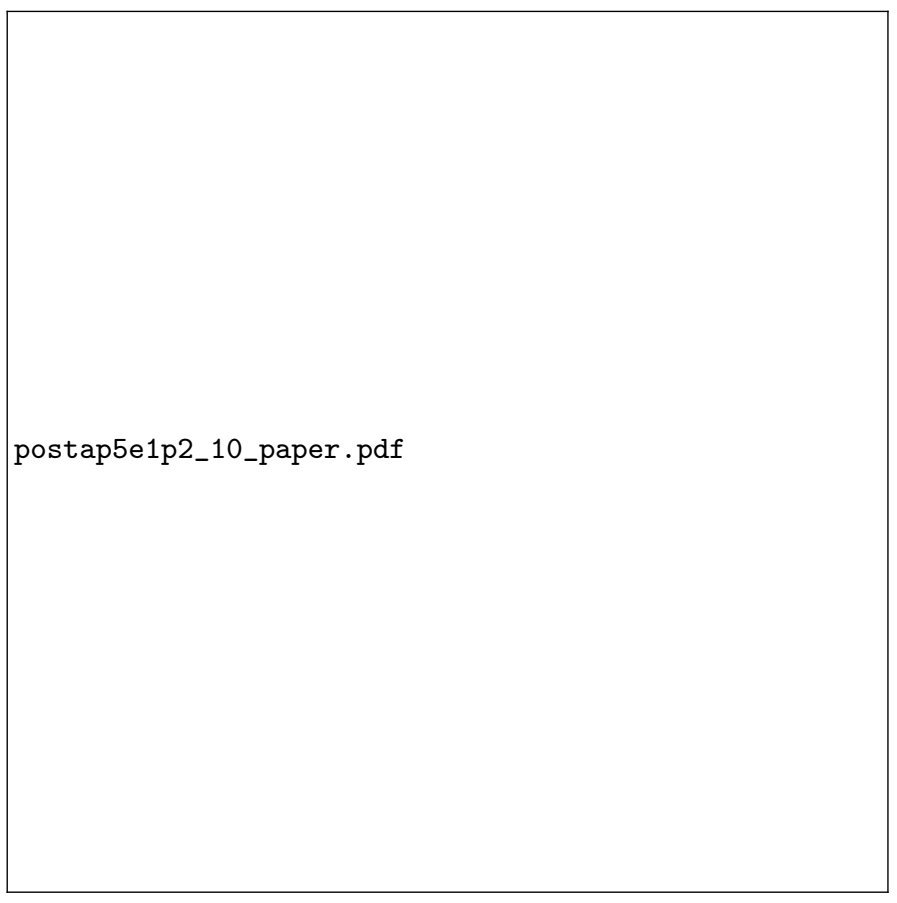

(b) $\mathscr{E}_{10}$ 


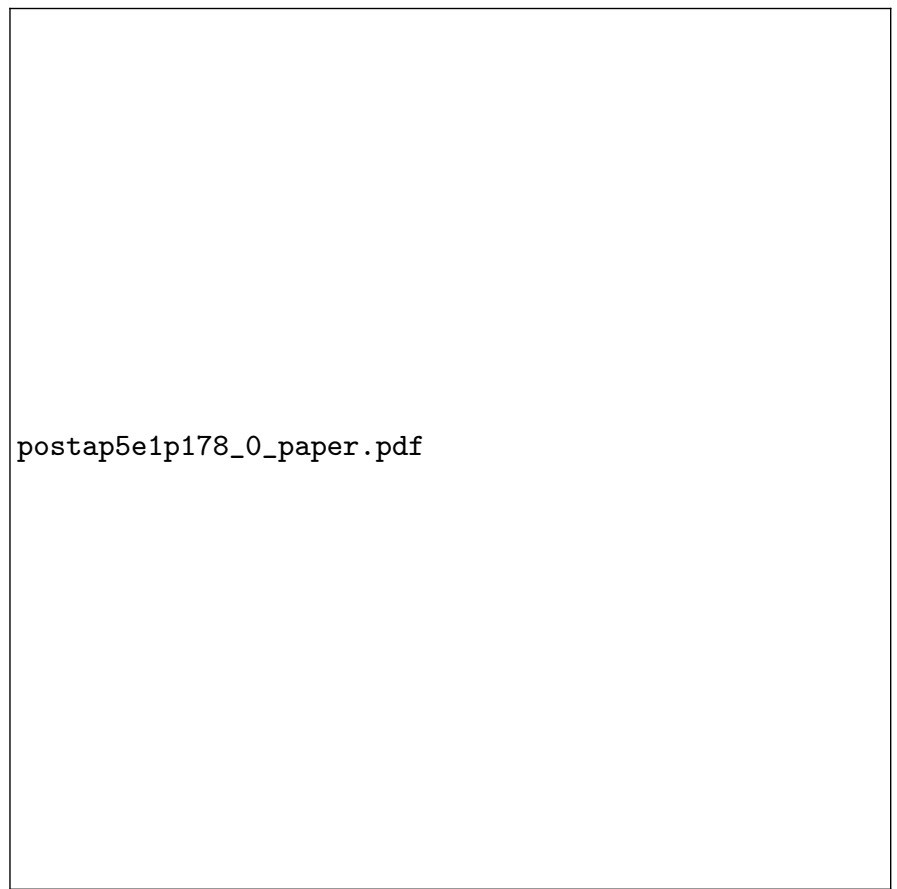

(a) $\mathscr{E}$

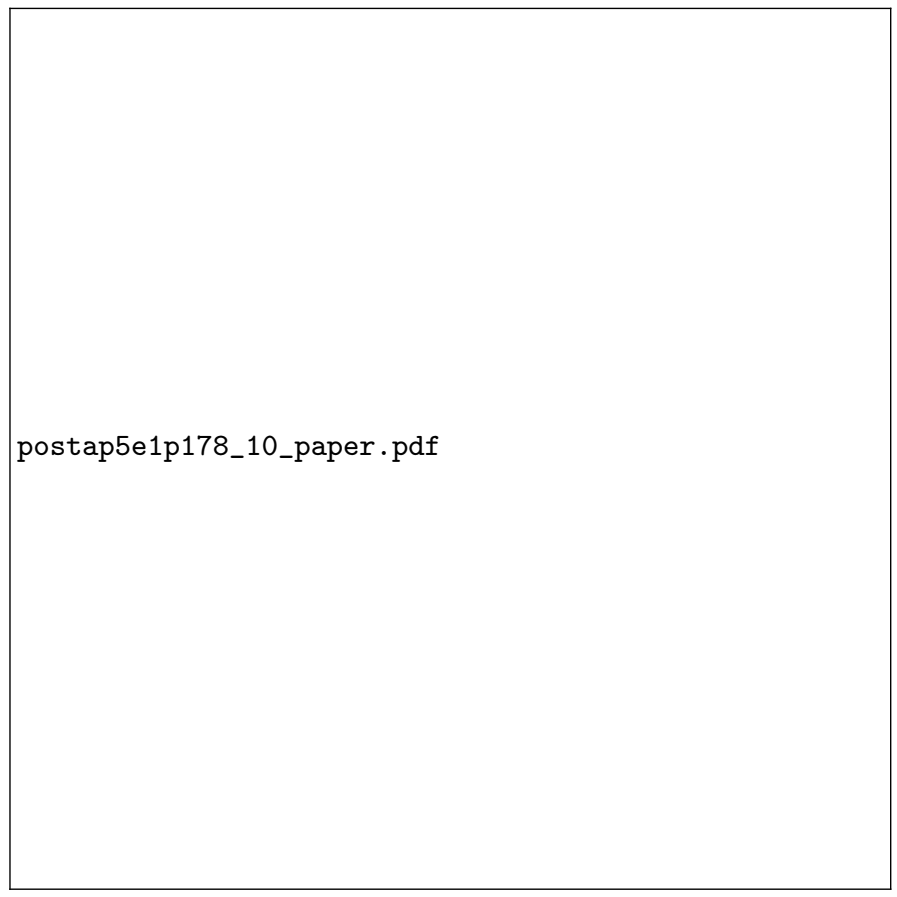

(b) $\mathscr{E}_{10}$

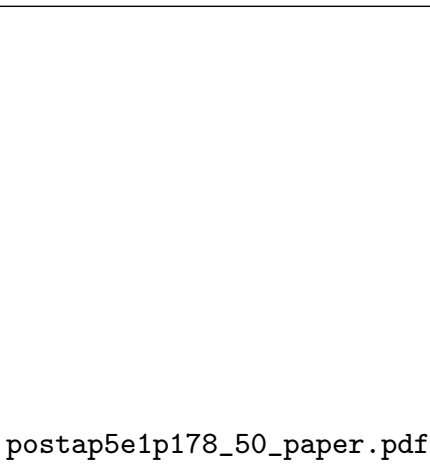

postap5e1p178_50_paper.pdf

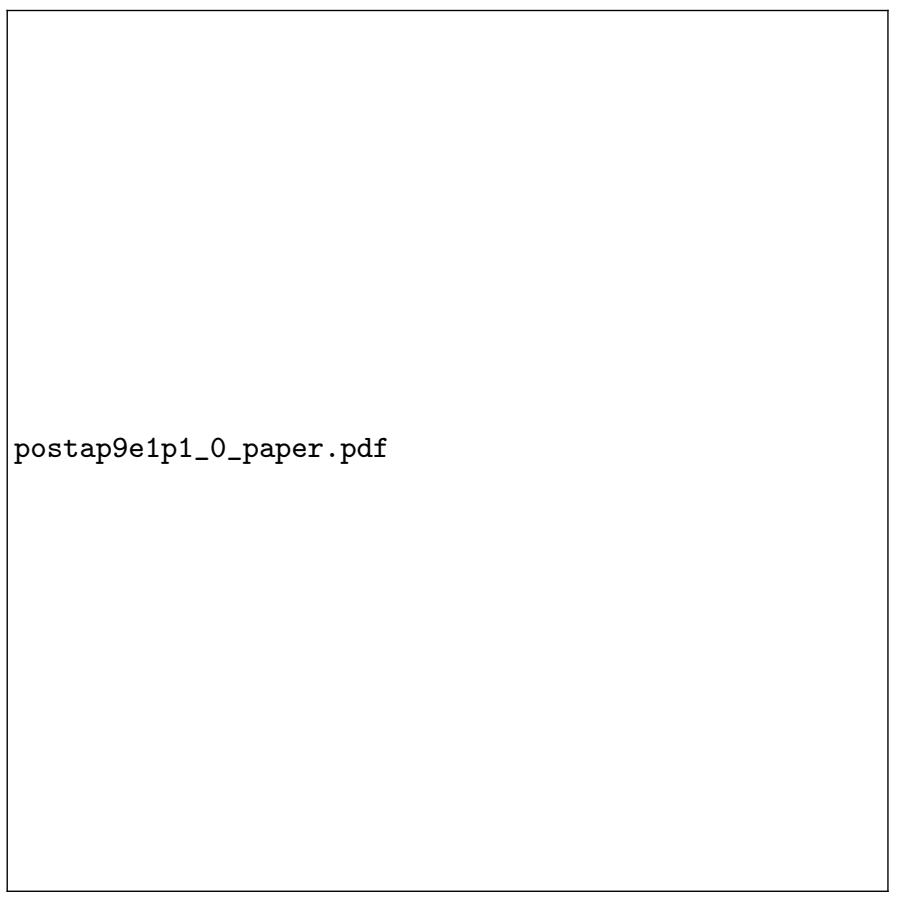

(a) $\mathscr{E}$

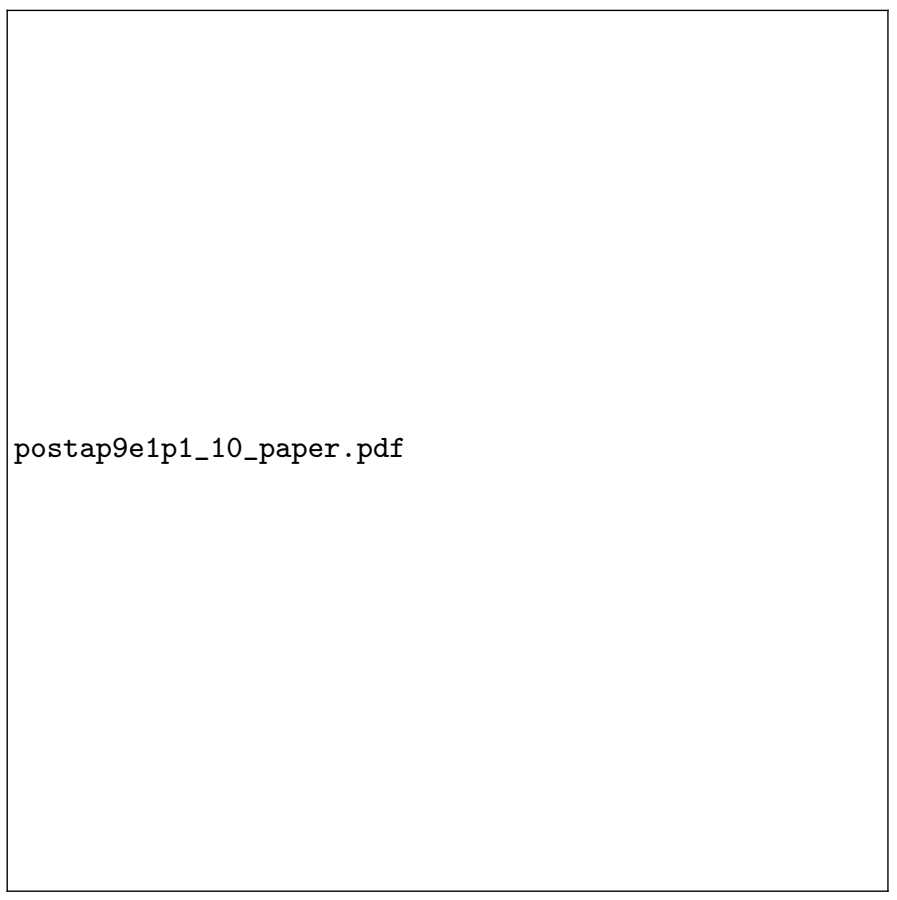

(b) $\mathscr{E}_{10}$ 


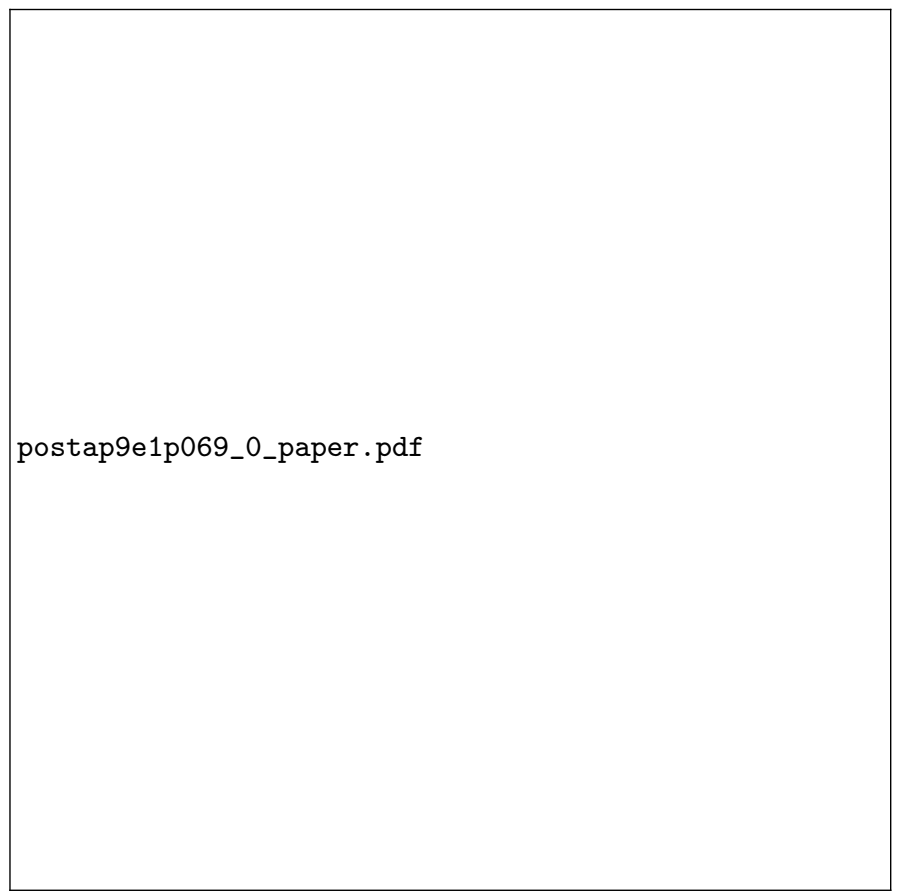

(a) $\mathscr{E}$

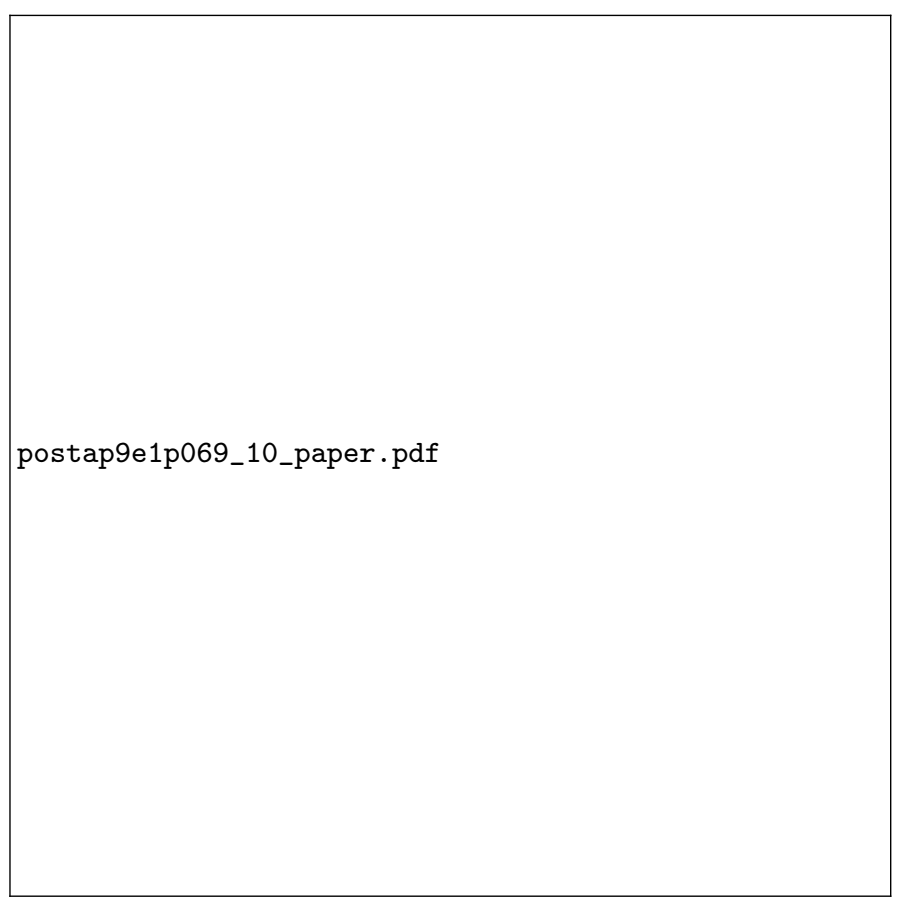

(b) $\mathscr{E}_{10}$

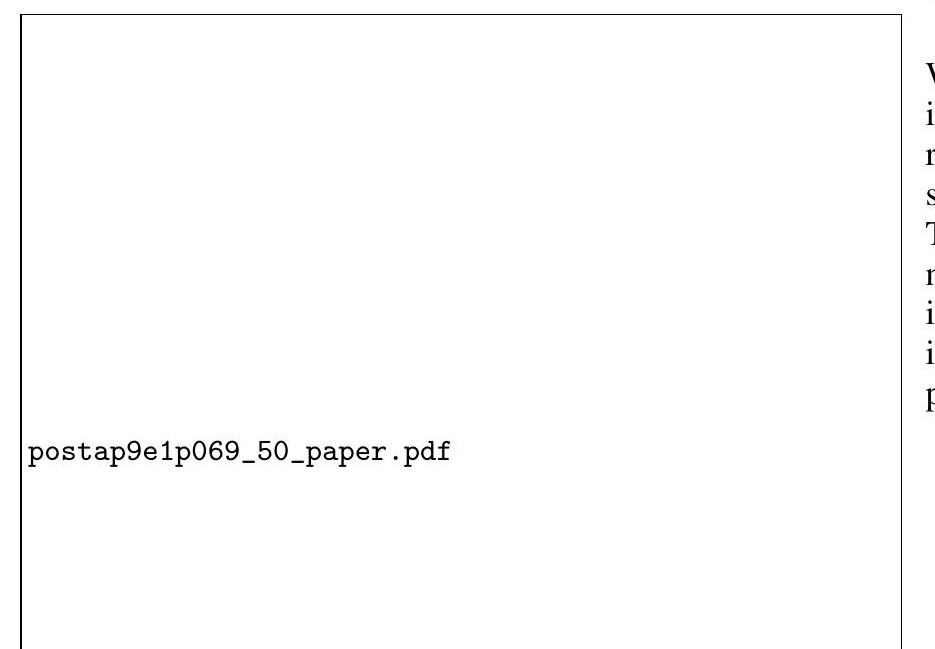

As it is challenging to fully characterize the forms displayed by the results, we focus on their common properties.

In every case, the distribution of $\theta_{e s c}$, at $\mathscr{E}$, is unintuitive and even, as for Fig. 16a, counter-intuitive. One might expect $\mathscr{E}$ to occur when the distance between the inner and outer boundaries is maximum, i.e. at $\theta_{e s c}=0$, yet here it is clearly a minimum. Appendix E displays heatmaps of $\theta_{\text {esc }}$ vs $n_{e s c}$, where this counter-intuitive behavior is also very visible. However, for every case studied, the distributions of $\theta_{\text {esc }}$ and $d_{\min }$ approach those that might be expected intuitively by $\mathscr{E}_{50}$. The holes that persist must relate to the structure of the global chaotic phase space, i.e. any trajectories within them belong to a disjoint set of trajectories within some KAM island. However, the same cannot be said for the dark bands within the heatmaps at $\mathscr{E}$. Furthermore it should be noted that the value of the maximum recorded 'count per bin' decreases by often more than an order of magnitude between the first and fiftieth escape event.

A common feature of most of the heatmaps at $\mathscr{E}$ is shown in greater detail within Fig. 22, where two distinctive band formations seem to merge into one. The variation in color is due to the variation of occupancy within different regions of the escape variable space, indicating that even within the set of coordinates at which the trajectory may escape, some are nonetheless more likely to be occupied than others. This may be analogous to the stickiness observed within the usual Poincaré sections.For some distributions collected, extreme variations of occupational density were observed, as clearly evidenced by the presence of the white points within, for example, Fig. 32b.

For values of elongation close to the critical value, the survival probability distribution is exponential. However, for values studied well above the critical value, the behaviors observed can be broken into two classes, those that clearly exponentially decrease immediately after $n_{\min }$, and those that exhibit exponential decrease after a transitory regime following $n_{\min }$. The prefactor $A$, of the linear semi-log fit of the survival probability distribution decreases by several orders of magnitude as $e$ approaches $e_{\text {crit }}$. This is expected as, during this approach, the probability of escape in finite time decreases to zero.

\section{DISCUSSION \& CONCLUSION.}

We introduced a new 2D billiard that exhibits mixed dynamics. As well as presenting complete Poincaré sections recurrence plots (RPs) were applied to the long-time dynamics resulting from unstable period- 2 orbits for different elongations. The study of a the RP features led to the identification of a new quantitative measure, that provided strong numerical evidence for the existence of a set of criticalelongations, shown in Fig. 8, marking the destruction of the final rotational transport barrier in the phase space, i.e. the two main portions of 


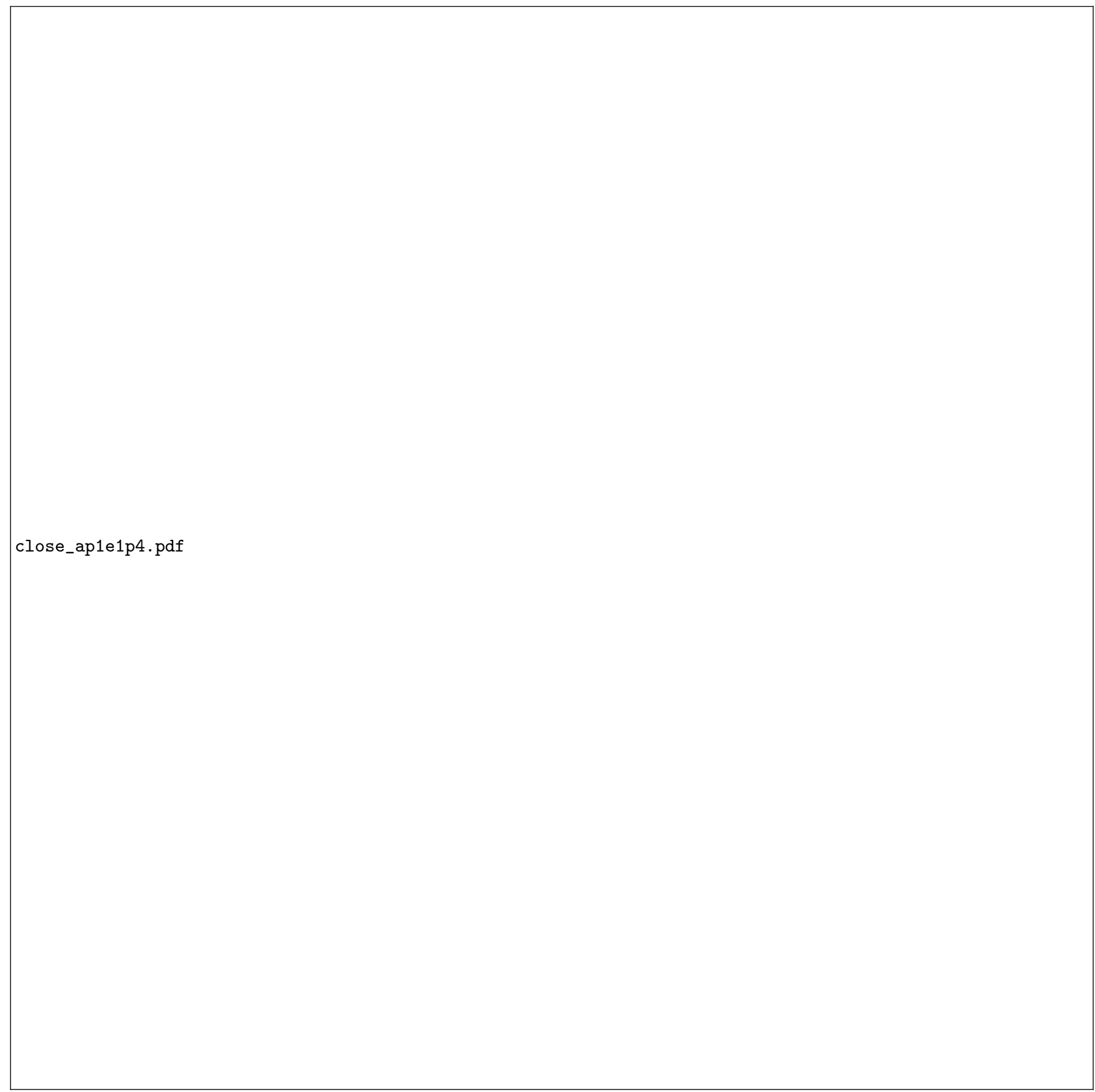

FIG. 22. Closeup of Fig. 10a highlighting the consistent 'merging' feature, present in most of the above heatmaps at $\mathscr{E}$.

the chaotic region of the phase space connect, causing a transition to global chaos.

The subfigures within Fig. 8 clearly show that the frequencies of the final destroyed rotational transport barriers correspond to different values of $\beta$. Contrary to the result obtained for the transition to global chaos in the standard map, we postulate that each critical value of elongation is directly associated to a different noble fraction, which defines the frequency of the final destroyed rotational transport barrier. The evidence suggests a connection between the set of noble numbers and the critical ellipse geometries, with deep consequences for the global structure of the chaotic phase space. To further explore this phenomenon, in the context of the system's symmetries, one would need a Poincaré first return map which, until now, is unobtainable by known means.

The endogenous escape event, $\mathscr{E}$, that signals the transition to global chaos, is illustrated in Fig. 9. It is so named to 
distinguish it from previously studied escape events that consider the first passage though some pre-defined hole in an open system. For example, survival probabilities of dynamical escape events have been previously studied in the case of 'open billiards', where a MUPO in a mushroom billiard, whose stem is replaced by a hole leads to a contribution to the survival probability proportional to $1 / t$ as $t \rightarrow \infty^{60}$. However, in this system, the 'holes' created are, unlike in open billiards, intrinsic to the phase space arising from the geometry considered. Furthermore the diffusive behavior of the reduced chaotic trajectory's components means that it would be probably incorrect to try to make an analogy with the previously studied MUPO escape probability distributions. It is, however, surprising that no power law distributions are at all observed as one would expect the presence of an infinite hierarchy of cantorous regular regions at the boundary between the two connected chaotic phase regions. The results obtained for different ellipse parameters, and therefore different times before the escape event, over which the survival probability is computed, means that one can be sure that the decaying exponential distributions are not masking some longer time power law distribution. Further results show that the distributions of both the angle of escape, and the distance of closest approach behave counter-intuitively at the moment of escape and for several iterations after, before relaxing to distributions one would expect for the chaotic behavior in a mixed dynamical system.

Initial investigations of the 3D version of the Iris billiard, following approaches used in Ref. ${ }^{61}$, show that an analogous critical geometric property discussed in this paper persists. It would therefore be natural to carry out a similar characterization of the 3D escape event $\mathscr{E}$. It is not clear how to obtain the observable escape features in 3 dimensions by similar arguments as employed here.

It would be interesting, and straightforward, to carry out a comparative study on the same phenomenon and resulting observables as studied here, in the context of the eccentric annular billiard ${ }^{51}$. If the transition to global chaos is again observed, it would then be possible to study the Poincaré map, which has already been obtained ${ }^{51}$, in order to identify the periodic orbits that become unstable at the critical perturbation value, via Greene's residue criterion ${ }^{53,62}$. Finally, it would be of great interest to perform superconducting microwave resonator experiments, such as those previously used to explore chaos-assisted dynamical tunneling ${ }^{27,28}$, with an Iris domain, or its desymmetrized version ${ }^{27}$. These could be carried out, for precisely machined geometries close to the critical values presented here. Such experiments could facilitate a detailed study of transport between the wave analogues of the parts of phase space that always, sometimes or never impinge upon the central ellipse.

\section{ACKNOWLEDGMENTS}

We thank Maxime Brunet for performing preliminary simulations of the Iris billiard.

\section{AIP PUBLISHING DATA SHARING POLICY}

The data that support the findings of this study are available from the corresponding author upon reasonable request.

\section{Appendix A: Derivation of conditions permitting rational $\omega_{1} / \omega_{2}$ orbits}

A rational $\omega_{1} / \omega_{2}$ orbit, where $\omega_{1}<\omega_{2}$, defines a $\omega_{2}$ polygon, whose radius of intersection of the enclosed caustic formed by its rotation through $\theta \in[0,2 \pi]$ follows:

$$
r_{\omega_{1}, \omega_{2}}=\left|\cos \left(\frac{\omega_{1} \pi}{\omega_{2}}\right)\right|
$$

Considering the parametric form of the ellipse, and applying simple geometric arguments returns the following condition for intersection,

$$
b^{2} \sin ^{2} \theta+a^{2} \cos ^{2} \theta-r_{\omega_{1}, \omega_{2}}^{2} \geq 0,
$$

which is true for all values of $\theta$ iff $r_{\omega_{1}, \omega_{2}}^{2} \leq a^{2}$. Conversely, there are no intersections for any value of $\theta$ when $r_{\omega_{1}, \omega_{2}}^{2} \geq b^{2}$. In the regime $a^{2} \leq r_{\omega_{1}, \omega_{2}}^{2} \leq b^{2}$, to determine if there is an intersection one must solve:

$$
b^{2} \sin ^{2} \theta+a^{2} \cos ^{2} \theta-r_{\omega_{1}, \omega_{2}}^{2}=0,
$$

returning:

$$
\sin ^{2} \theta=\frac{r_{\omega_{1}, \omega_{2}}^{2}-a^{2}}{b^{2}-a^{2}}
$$

Therefore the conditions for intersection with the inner ellipse are therefore met by:

$$
|\theta-\pi / 2| \leq \arccos \sqrt{\frac{r_{\omega_{1}, \omega_{2}}^{2}-a^{2}}{b^{2}-a^{2}}},
$$

or, by the symmetry of the system,

$$
|\theta-3 \pi / 2| \leq \arccos \sqrt{\frac{r_{\omega_{1}, \omega_{2}}^{2}-a^{2}}{b^{2}-a^{2}}},
$$


In order for a rational $\omega_{1} / \omega_{2}$ orbit to exist, one must avoid intersections for all $\theta+2 \pi n / \omega_{2} \forall n \in\left[0, . ., \omega_{2}-1\right]$. Therefore, the following condition must be met:

$$
\arccos \sqrt{\frac{r_{\omega_{1}, \omega_{2}}^{2}-a^{2}}{b^{2}-a^{2}}}<\frac{\pi}{\kappa\left(\omega_{2}\right) \omega_{2}}, \omega_{2} \in\{3,4,5 \ldots\}
$$

where:

$$
\kappa\left(\omega_{2}\right)=1+\omega_{2} \bmod 2 .
$$

Equation (A7) may be finally rearranged to give:

$$
\frac{b}{a} \geq e_{\min }=\left[\frac{1}{\cos ^{2}\left(\pi / \kappa\left(\omega_{2}\right) \omega_{2}\right)}\left(\frac{r_{\omega_{1}, \omega_{2}}^{2}}{a^{2}}-1\right)+1\right]^{1 / 2}
$$

\section{Appendix B: Stability analysis of Period 2 orbits}

Each orbit within the billiard is an ensemble of alternating straight lines and reflections. To describe the effect of changing the initial conditions on a trajectory of $n$ segments we construct a stability matrix, $\mathbf{M}_{n}$, such that:

$$
\left(\begin{array}{c}
\delta \theta_{n} \\
\delta \beta_{n}
\end{array}\right)=\mathbf{M}_{n}\left(\begin{array}{c}
\delta \theta_{0} \\
\delta \beta_{0}
\end{array}\right)
$$

Slight changes, $\left(\delta \theta_{0}, \delta \beta_{0}\right)$, made to the initial position perpendicular to the direction of motion and to the initial direction, respectively, at $\left(\theta_{0}, \beta_{0}\right)$, through the operation of $\mathbf{M}_{n}$, produce the resultant deviations $\left(\delta \theta_{n}, \delta \beta_{n}\right) . \mathbf{M}_{n}$ is computed as the product of matrices describing the effect of the deviations to each straight line segment and reflection of a trajectory resulting from a slight change in its initial conditions:

$$
\mathbf{M}_{n}=\prod_{i=0}^{n}\left(\begin{array}{ll}
1 & l_{i} \\
0 & 1
\end{array}\right) \cdot\left(\begin{array}{cc}
-1 & 0 \\
\frac{-2}{R_{i} \cos \beta_{i}} & -1
\end{array}\right)
$$

where $l_{i}$ is the length of the $i^{t h}$ segment and $R_{i}$ is the radius of curvature of the, inner or outer, boundary at the $i^{t h}$ collision. Since the system is conservative, the mapping describing the evolution of the trajectory is area preserving, i.e.

$$
\operatorname{Det} \mathbf{M}_{\mathrm{n}}=1 .
$$

Eigenvalues of the stability matrix, and therefore the properties of the trajectory, depend only upon its trace. To best obtain the analytical properties of the trajectory, its residue, R, as defined by Greene ${ }^{53}$, is used, where:

$$
R=\frac{1}{4}\left(2-\operatorname{Tr} \mathbf{M}_{\mathrm{n}}\right)
$$

The values of $R$, determined by the value of $\operatorname{Tr} \mathbf{M}_{\mathrm{n}}$, characterize the analytic properties of a trajectory as follows:

$$
\begin{cases}R<0 & \text { Hyperbolic } \\ R=0, & \text { Marginally Stable } \\ 0<R<1 & \text { Stable. } \\ R>1 & \text { Reflection Hyperbolic }\end{cases}
$$

Hyperbolic trajectories correspond to $\mathbf{M}_{n}$ having real eigenvalues that are positive (or negative in the reflection case). They always undergo significant deviation due to the finite limits of numerical precision. Marginally stable trajectories correspond to eigenvalues of \pm 1 . The stable case yields complex eigenvalues with magnitude unity. Two stable trajectories, with slightly different initial conditions, undergo a linear deviation over time.

The trace of the stability matrix of a single period 2 orbit in the direction of the ellipse's semi-minor axis is:

$$
\operatorname{Tr}\left(\mathbf{M}_{2}^{\mathrm{a}}\right)=\frac{4 \mathrm{~b}\left(\mathrm{e}^{2}-1\right)}{\mathrm{e}^{3}}+\frac{4}{\mathrm{e}^{2}}-2 .
$$

$\left|\operatorname{Tr}\left(\mathbf{M}_{2}^{\mathrm{a}}\right)\right| \leq 2 \quad \forall \mathrm{a}$, e. Therefore the residue of the orbit always indicates stability $(0<R<1)$.

Along the direction of the semi-major axis, the trace otherwise returns:

$$
\operatorname{Tr}\left(\mathbf{M}_{2}^{\mathrm{b}}\right)=-4 \mathrm{~b}\left(\mathrm{e}^{2}-1\right)+4 \mathrm{e}^{2}-2 .
$$

In this case the orbit is always hyperbolic $(R<0)$, except for where it yields marginal stability $(R=0)$ in the limiting cases, $a=b$ and $b=1$. (If $a=b$, i.e. for a circular inner scatterer, all trajectories from every initial condition are either periodic or quasi-periodic, with conserved angular momentum. If $b=1$, the system becomes two separate crescents). As time tends to infinity, the stable orbit will continue unchanged; while the unstable orbit, knocked off course by numerical imprecision, will explore the accessible chaotic sea.

\section{Appendix C: Recurrence Quantification Analysis as indicators of stickiness}

To study the structures presented by RPs, several measures ${ }^{63}$, known as Recurrence Quantification Analysis (RQA), are already in use. We will focus specifically on two in the context of the chaotic dynamics of the Iris Billiard. The first measure is the recurrence rate (RR), defined as the percentage of black points in an RP: 


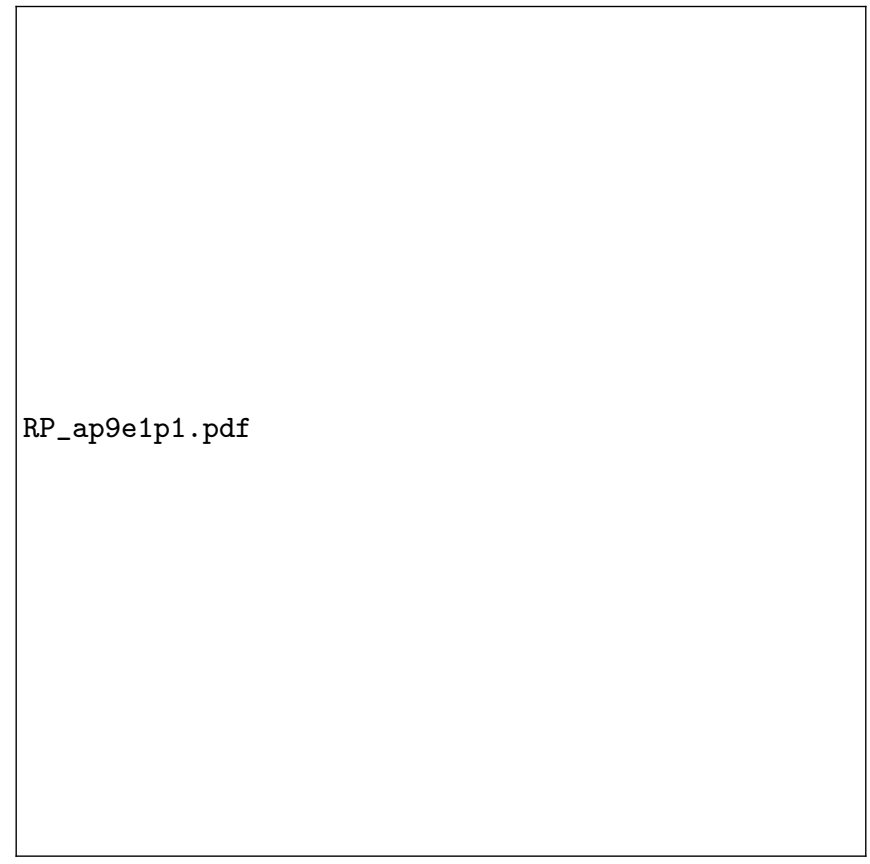

FIG. 23. RP of a $2 \times 10^{4}$ long chaotic trajectory, launched from the unstable period-two orbit, for $a=0.9, e=1.1 . R R_{\text {crit }}=0.1 . D E_{c r i t}=$ 0.3 . Three separate regions of interest are selected and marked as I, II and III.

$$
R R(\varepsilon)=\frac{1}{N^{2}} \sum_{i, j=1}^{N} \mathbf{R}_{i, j}(\varepsilon)
$$

This may be better understood as the 'sparsity' of the $N \times N$ binary matrix under consideration. In the limit $N \rightarrow \infty, R R$ is the probability that a state recurs to its phase neighbourhood, as demarked by $\varepsilon$.

The second measure is based on the distribution of diagonal lines present within the $R P$ :

$$
P(\varepsilon, l)=\sum_{i, j=1}^{N}\left(1-\mathbf{R}_{i-1, j-1}\right)\left(1-\mathbf{R}_{i+l, j+l}\right) \prod_{k=0}^{l-1} \mathbf{R}_{i+k, j+k}
$$

Recurrence plots principally show diagonal lines for periodic and quasiperiodic orbits, as one of length $l$ shows that a segment of a trajectory is close to another segment from a different time, for $l$ iterations. The trajectory's determinism (DET), is defined as the percentage of black points belonging to a diagonal line of at least $l_{\min }$. The ratio of recurrence points that form a diagonal line, of at least length $l_{\min }$, to all the recurrence points, therefore, provides a measure of how deterministic (predictable) the trajectory is,

$$
D E T=\frac{\sum_{l=l_{\min }}^{N} l P(\varepsilon, l)}{\sum_{l=1}^{N} l P(\varepsilon, l)} .
$$

In what follows, $l_{\min }=3$. For all periodic orbits $D E T=1$. To apply these measures as indicators of stickiness within the billiard dynamics, windows of size $l_{w}=200$ will be overlayed the original recurrence plot. $R Q A$ will be applied to each window, and the evolution of the above introduced variables will serve as indicators of dynamical transitions.

An example RP applied to a chaotic orbit of length $2 \times 10^{4}$ is shown. The measures introduced above are applied as a particularly sensitive measurement of intra-chaotic dynamical transitions. Fig 23 shows the RP of a long, chaotic orbit for $a=0.9, e=1.1$. The trajectory is then analysed by applying the previously introduced RQA measures to moving windows of length $w=200$. The selected RQA measures $(R R \&$ $D E T)$ are monitored with respect to time. When the chaotic trajectory encounters a sticky region, the $R R$ significantly changes as its evolution becomes much more regular. Three examples are highlighted in Fig 23 as Domains I, II and III. These correspond to regions where both $R R$ and $D E T$ surpass $R R_{\text {crit }}$ and $D E T_{\text {crit }}$, defined as 0.1 and 0.3 respectively. These values were chosen for the purpose of demonstrating the presence and detection of stickiness for one specific geometry, and would have to be reevaluated for each different geometry considered. Domain III is of particular interest as it corresponds a high deviation for only the $D E T$ measure, but not for $R R$. Figs. $2425 \& 26$ show the phase occupation of the sticky orbits observed by the $R R$ and $D E T$ measures.

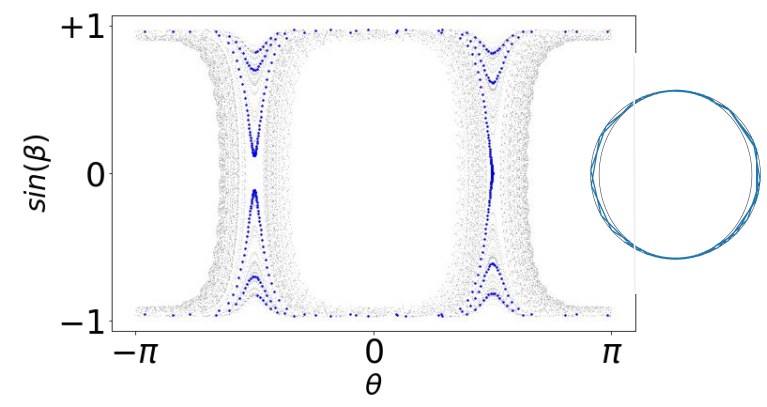

FIG. 24. Domain I, $i \in 352,1100$ : Rotational motion, of different frequencies,

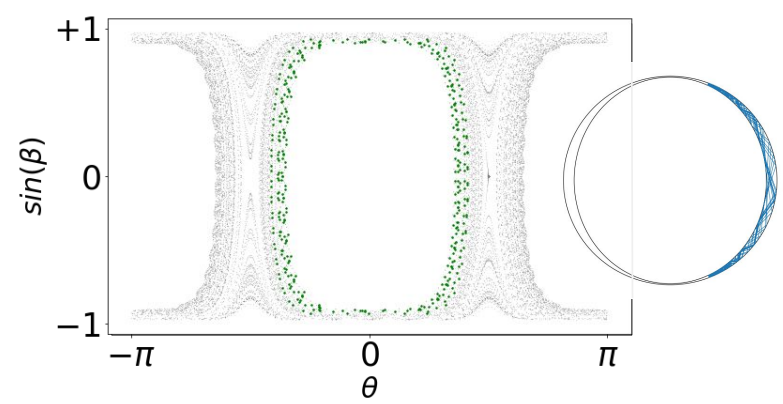

FIG. 25. Domain II, $i \in$ 9420,5200: Librational diffusion. 


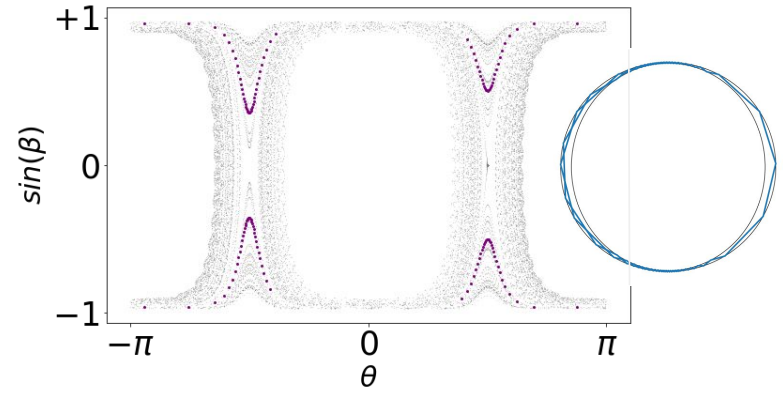

FIG. 26. Domain III, $i \in 9400,9540$ Short rotational motion.

\section{Appendix D: Derivation of $\theta_{e s c}$ and $d_{\min }$}

Let $\hat{u}$ be the unit vector specifying the trajectory. For a nonintersecting trajectory, there is a point of closest approach on the ellipse, $r_{e}=\left(x_{e}, y_{e}\right)$, whose tangent is parallel to $\hat{u}$, see Fig. 9. Thus

$$
\frac{d y}{d x}=-\frac{x b^{2}}{y a^{2}}=\frac{u_{y}}{u_{x}} .
$$

Substituting for $\mathrm{x}$ in the ellipse equation, Eq. 3, returns

$$
y^{2}=\frac{b^{4}}{\left(a u_{y} / u_{x}\right)^{2}+b^{2}} .
$$

Since there are two points on the ellipse whose tangents are parallel to $\hat{u}$, we obtain two solutions: $\left(x_{e}, y_{e}\right)$ and $\left(x_{e 1}, y_{e 1}\right)$.

Applying simple geometric reasoning, as shown in Fig. 27 gives the minimum distance of approach as:

$$
d_{\min }^{2}=\left|\boldsymbol{r}_{\mathbf{1}}-\mathbf{r}_{\mathbf{e} / \mathbf{e} \mathbf{1}}\right|^{2}-\left[\hat{\mathbf{u}} \cdot\left(\mathbf{r}_{\mathbf{1}}-\mathbf{r}_{\mathbf{e} / \mathbf{e} \mathbf{1}}\right)\right]^{2} .
$$

The sought after solution is the one that returns the smallest value of $d_{\min }$, from which one may directly obtain the associated value of $\theta_{\text {esc }}$.

\section{Appendix E: Heat maps of $\theta_{e s c}$ vs $n_{e s c}$}

The heatmaps presented in Figs. 28, 29, 30, 31, 32 and 33 show the joint distribution of the number of iterations before escape, $n$, against the angle of escape, $\theta_{\text {esc }}$, at the first escape event (i.e. the first miss), the tenth miss and the fiftieth miss. These results show the high sensitivity of the escape event to the billiard geometry. An example is given in Figs. 30 (a) and 31 (b), where there is only a $2 \%$ difference in the elongation. Furthermore, for every case studied, there is a clear averaging effect on the distribution of the observable as the number of ellipse misses increases, whereas the statistics of the first escape show bands of non-occupation, that can be related to the structures observed in Sec. VB. 


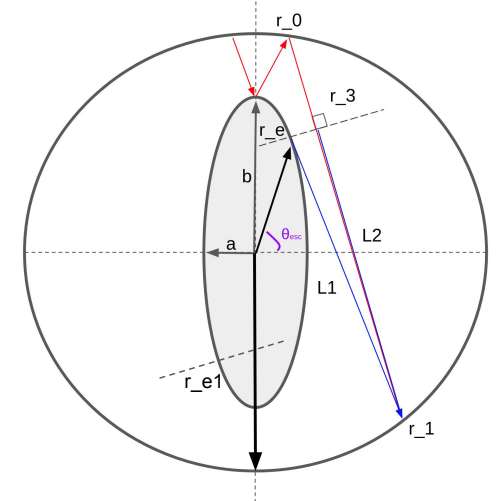

(a)

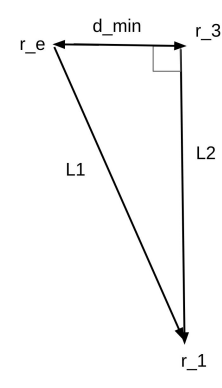

(b)

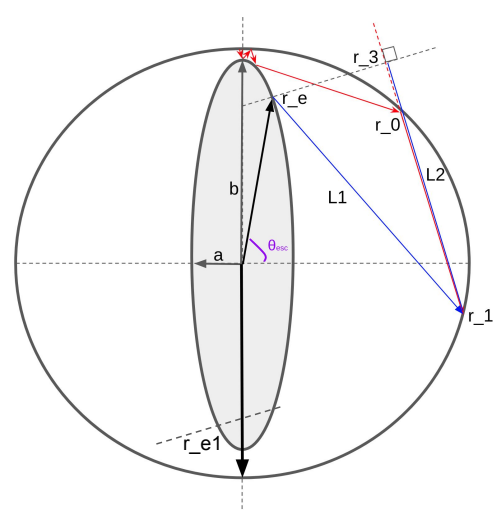

(c)

FIG. 27. Figures showing the geometric construction used to obtain $\theta_{e s c}$ and $d_{\text {min }}$. (a) A 'real' case. Where $d_{\text {min }}$ is obtained via reasoning shown in (b): $L 2=\hat{\mathbf{u}} \cdot\left(\mathbf{r}_{\mathbf{1}}-\mathbf{r}_{\mathbf{e}}\right)$. (c) A 'virtual' case, where the escaping trajectory is only parallel to the ellipse at a constructed point extended beyond the Iris boundary. This situation is most likely for billiards close to the crescent configuration.

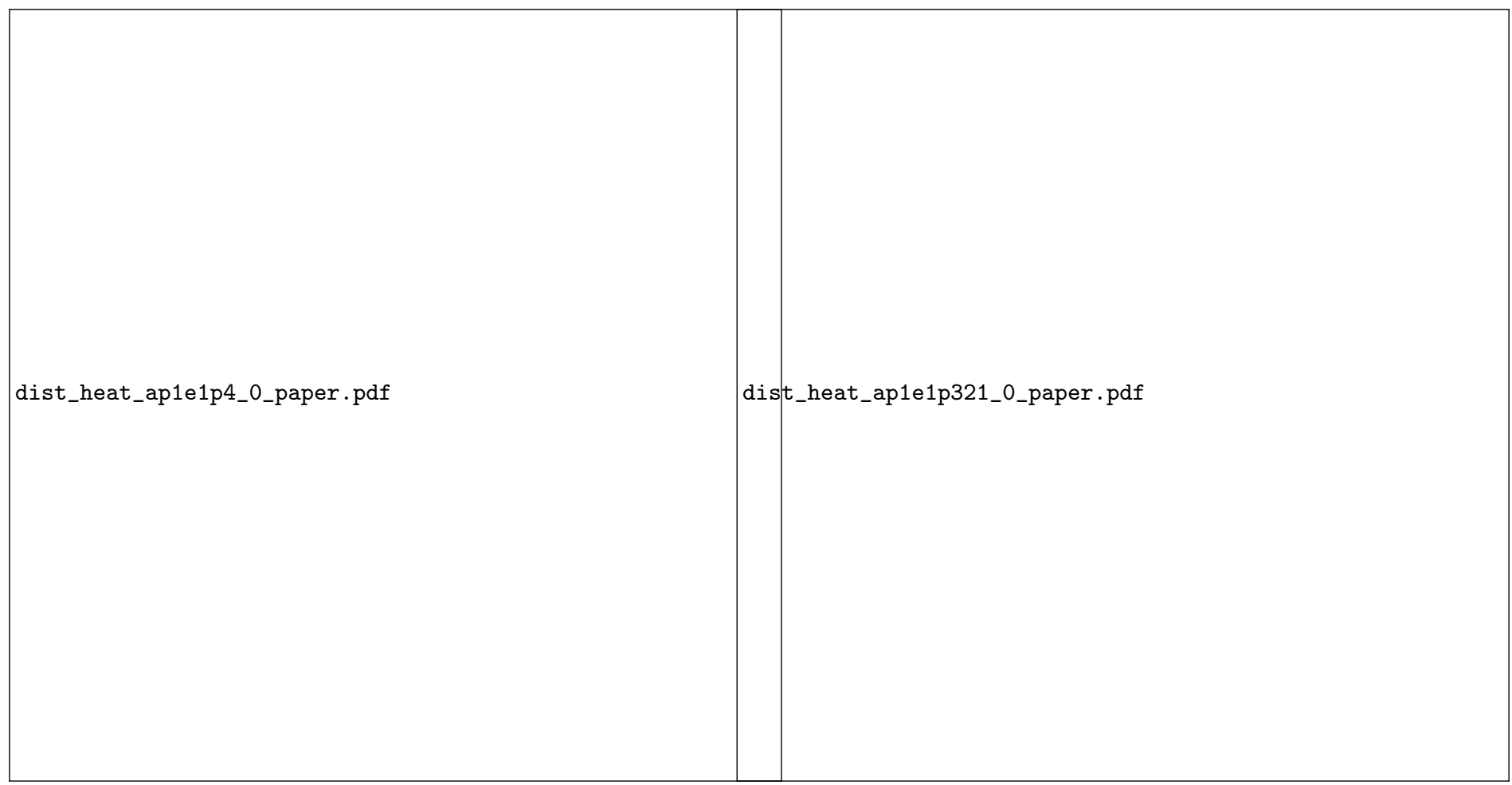

(a) $\mathscr{E}$

(a) $\mathscr{E}$ 


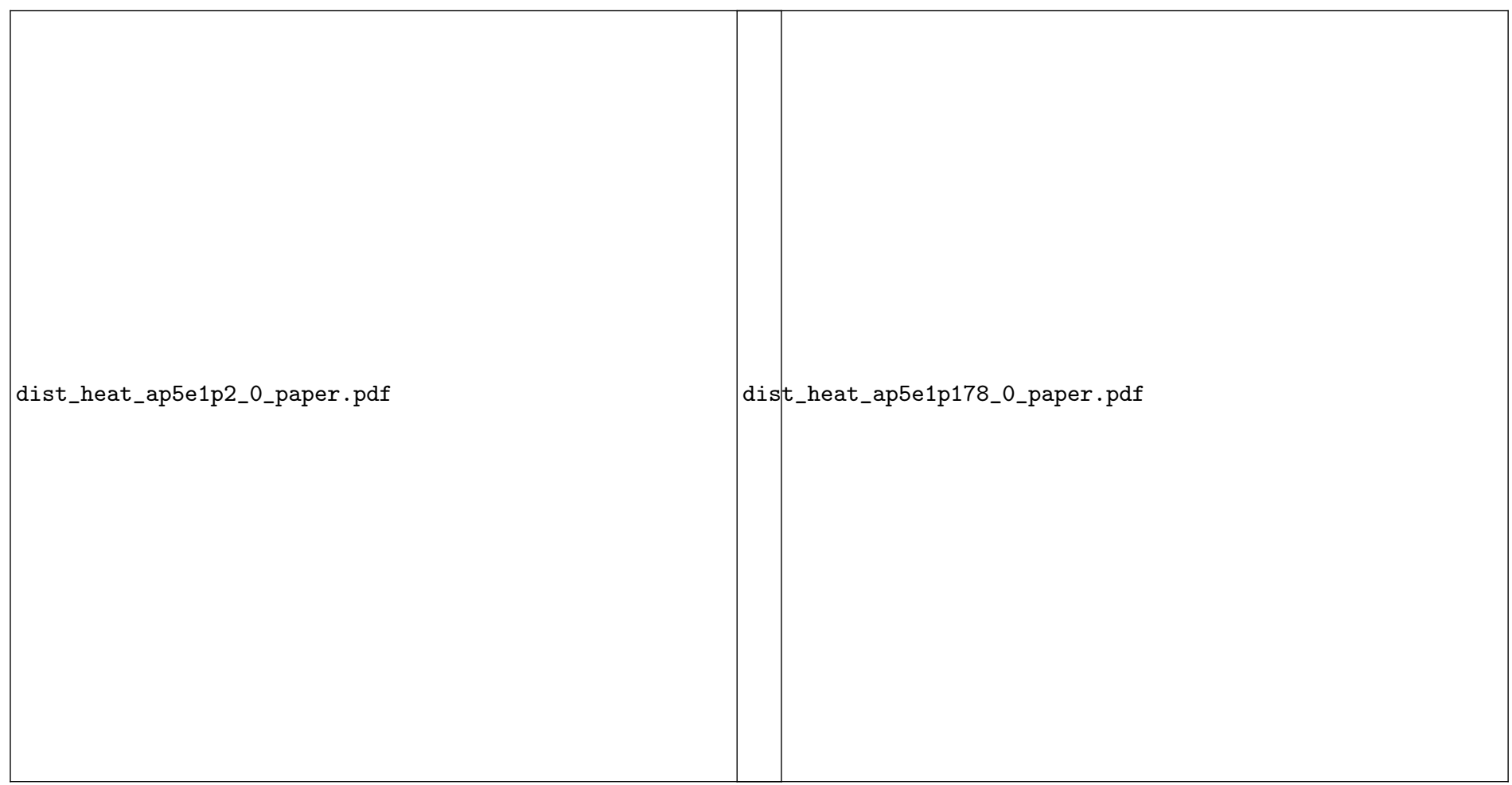

(a) $\mathscr{E}$

(a) $\mathscr{E}$

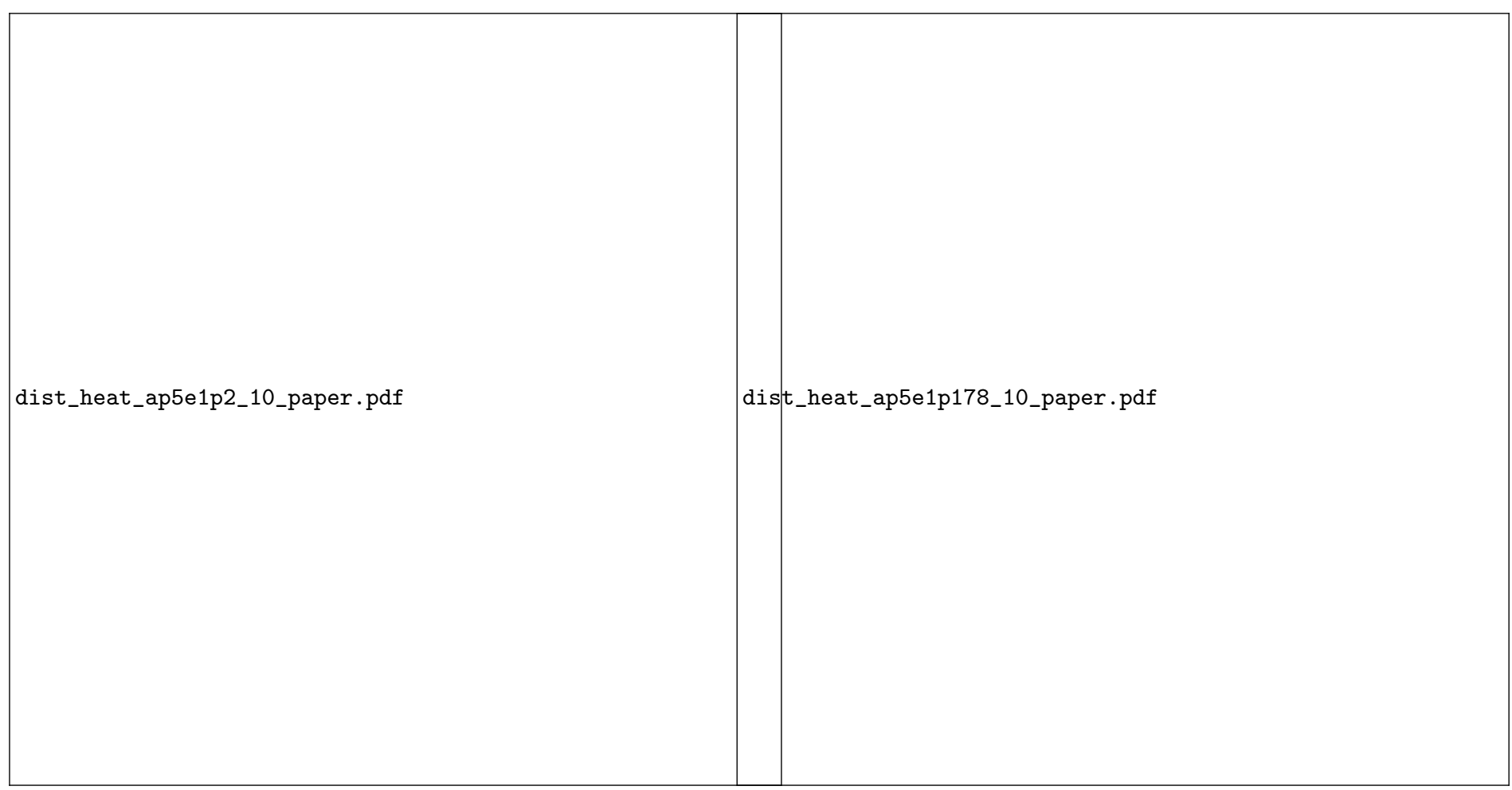

(b) $\mathscr{E}_{10}$

(b) $\mathscr{E}_{10}$

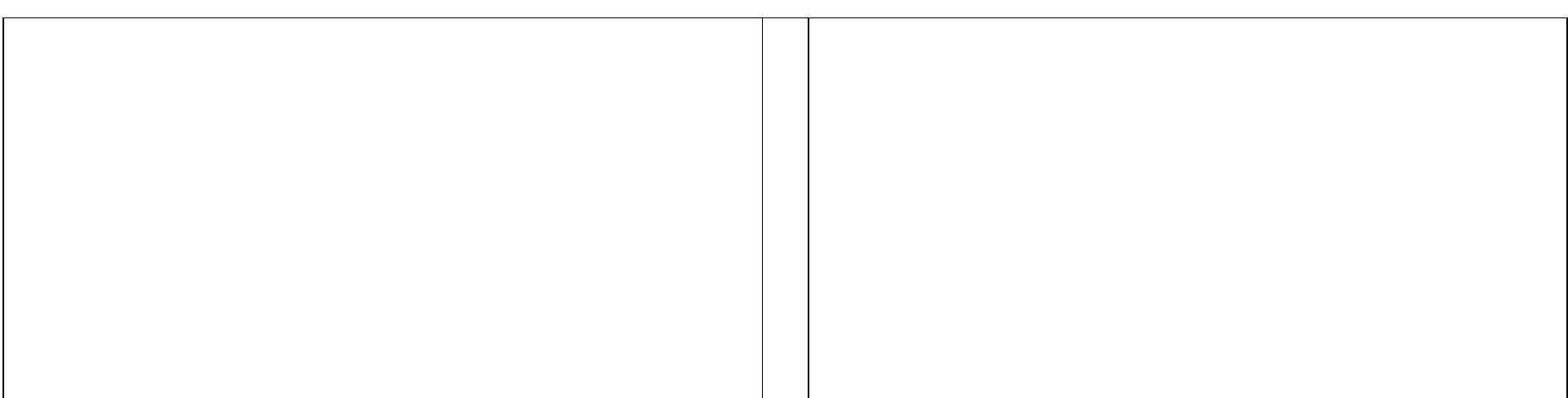




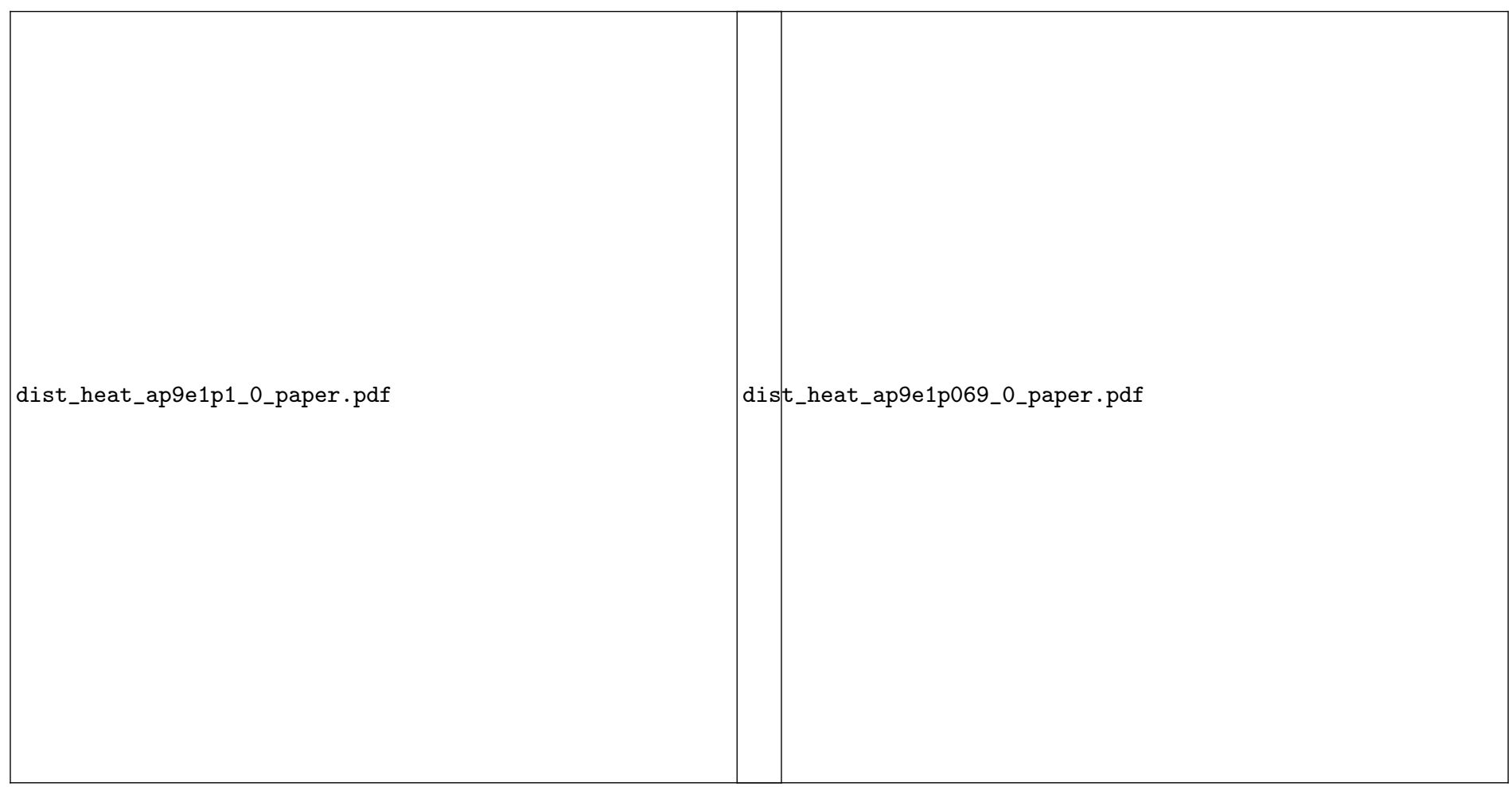

(a) $\mathscr{E}$

(a) $\mathscr{E}$

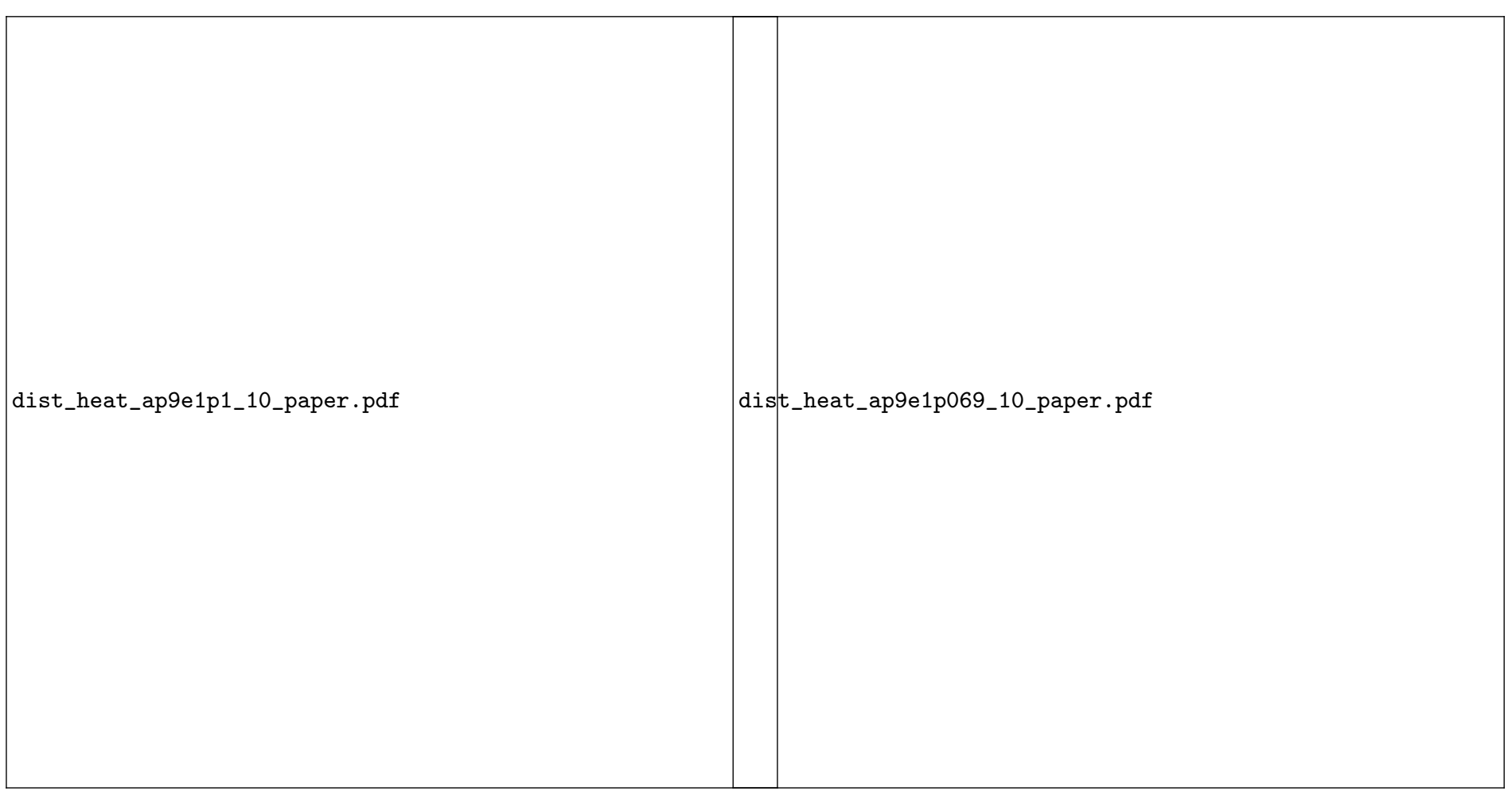

(b) $\mathscr{E}_{10}$

(b) $\mathscr{E}_{10}$

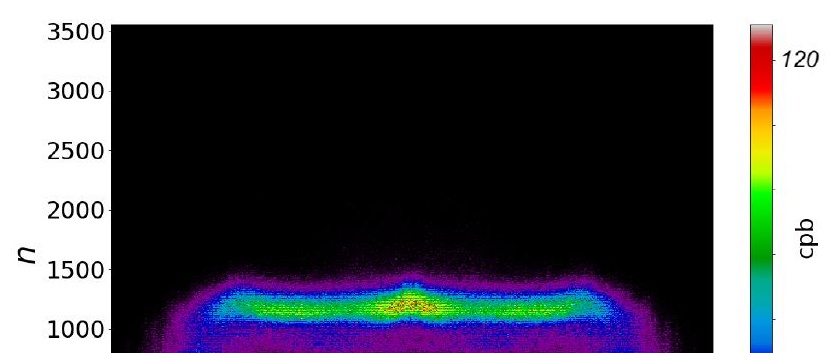




\section{Appendix F: Box counting dimension around critical geometries}

We characterize the chaos/order boundary by considering its box-counting dimension. This is done by defining $N_{B}(\varepsilon)$ as the number of cells needed to cover the chaos border at given value of $\varepsilon$. We understand dimension to mean how much space a set occupies near each of its points.

The idea of a 'measurement' of a set at scale, $\varepsilon$, defined by $L_{\infty}$., is fundamental. For each $\varepsilon$, one measures the set in a way that detects irregularities of size $\Delta \varepsilon$. Ultimately we want to know how these measurements behave as $\varepsilon \rightarrow 0^{64}$.

Defining the chaos border as the subset, $B$, for $\varepsilon>0$, we also define the smallest number of sets of maximum diameter $\varepsilon$ as $N_{B}(\varepsilon)$. The dimension of $B$ reflects the way in which $N_{B}(\varepsilon)$ grows as $\varepsilon \rightarrow 0$. If $N_{B}(\varepsilon)$ even approximately behaves as a power law, i.e.

$$
N_{B}(\varepsilon) \simeq c \varepsilon^{-D}, \forall c, D \geq 0
$$

where c is some constant, $B$ is then said to have a 'boxcounting dimension' $D$. This is solved via:

$$
\begin{aligned}
& \log N_{B}(\varepsilon) \simeq \log (c)-D \log (\varepsilon) \\
& \therefore D \simeq \frac{-\log N_{B}(\varepsilon)}{\log (\varepsilon)}+\frac{\log (c)}{\log (\varepsilon)}
\end{aligned}
$$

allowing us to obtain $D$, in the limit, as:

$$
D=\lim _{\varepsilon \rightarrow 0} \frac{\log N_{B}(\varepsilon)}{\log (1 / \varepsilon)} .
$$

The second term disappears in the limit. The chaos border subset, $B$, is defined as the set of 'border cells', i.e. that have been visited but have at least one 'empty' neighbouring cell alongside. The chaotic orbit must have visited a border cell at least once, meaning that some part of the phase space contained within is the chaotic component. However, it must be recognized that, in all probability, the whole cell is not filled by the chaotic phase component.

To determine the fractal dimension, grid cells between $L=$ 500 and $L=1500$ were used. The length of each chaotic orbit was $2 \times 10^{9}$, to ensure a large number of counts per cell, even for larger values of $L$. Using short trajectories to fill the chaotic set results in an anomalous deviation of the border cell count with number of cells $L$ from the expected power law. This is because the sparsity of points recorded within the chaotic phase portion leads to the misidentification of border cells where, in fact, there are none.

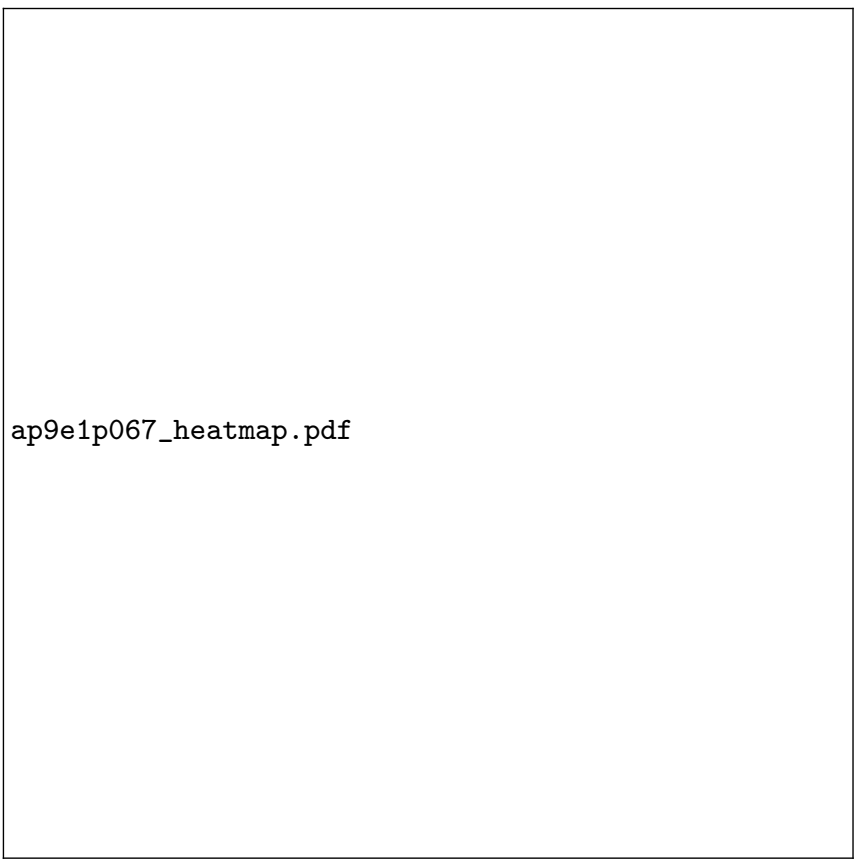

FIG. 34. Heatmap of the reduced chaotic portion of the phase space, prior to global connection, filled by a $2 \times 10^{9}$ long trajectory launched from the unstable period-two orbit. $L=300, a=0.9, e=$ 1.067. All trajectories launched from any initial condition, within this chaotic set, will obey the symbolic parity condition for all time. 


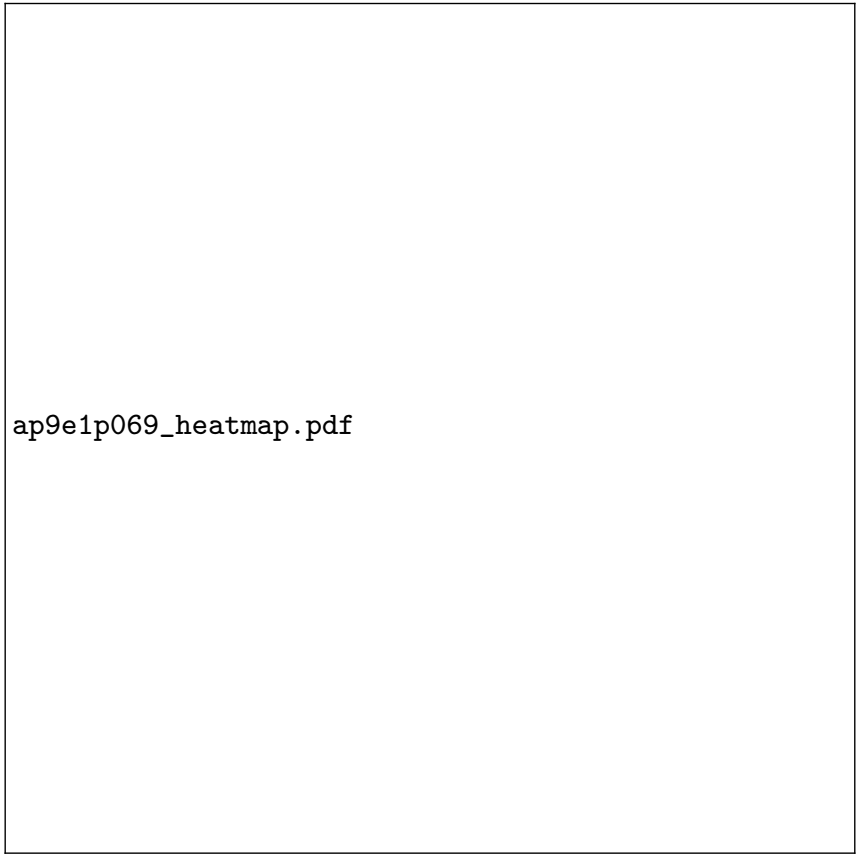

FIG. 35. Heatmap of chaotic potion of the phase space post global connection. $L=300, a=0.9, e=1.069$, filled by a $2 \times 10^{9}$ long trajectory launched from the unstable period-two orbit. In this case, all chaotic trajectories, may diffuse in and out of the region that obeys the parity condition. The closer to the critical elongation one approaches, from above, the more time passes between the passages between the two dynamical regime. For values of elongation that approach the critical limit from above, the rate of diffusion lessens.

The fractal dimension of the chaos/order boundary at values of elongation above and below the critical values measured by trajectories of length $N=2 \times 10^{9}$ are presented in Tables II and II.

\begin{tabular}{l|l|l}
$a$ & $e$ & $D$ \\
\hline 0.1 & 1.317 & $1.70 \pm 1 \times 10^{-2}$ \\
0.5 & 1.175 & $1.62 \pm 2 \times 10^{-3}$ \\
0.9 & 1.067 & $1.80 \pm 7 \times 10^{-3}$
\end{tabular}

TABLE I. Table the fractal dimensions of the border of the chaotic component, for chosen elongations under the critical set.

\begin{tabular}{l|l|l}
$a$ & $e$ & $D$ \\
\hline 0.1 & 1.321 & $1.63 \pm 1 \times 10^{-2}$ \\
0.5 & 1.178 & $1.53 \pm 3 \times 10^{-3}$ \\
0.9 & 1.069 & $1.95 \pm 1 \times 10^{-2}$
\end{tabular}

TABLE II. Table of the evolution of the fractal dimensions for the border of the chaotic component, for chosen elongations above the critical set.

Both Figs. 34 and 35 show very clearly the presence of cantori (destroyed remnants of perturbed robust transport barriers) above and below the critical destroyed barrier, that must correspond to the the remains of previously destroyed (but nonetheless robust) rotational transport barriers. The marked presence of these structures is what causes the fractal dimension of the chaos order border for $a=0.9$ to be very high, indeed higher than those measured measured for other ellipse parameters, and close to the upper dimensional limit, 2.

${ }^{1} \mathrm{~S}$. Tabachnikov, Geometry and billiards (American Mathematical Soc., 2005).

${ }^{2}$ L. A. Bunimovich and C. P. Dettmann, "Open circular billiards and the riemann hypothesis," Physical Review Letters 94 (2005), 10.1103/physrevlett.94.100201.

${ }^{3}$ M. V. Berry, "Regularity and chaos in classical mechanics, illustrated by three deformations of a circular billiard," European Journal of Physics 2, 91-102 (1981).

${ }^{4}$ R. Garcia, "Elliptic billiards and ellipses associated to the 3-periodic orbits," The American Mathematical Monthly 126, 491-504 (2019).

${ }^{5}$ M. A. Bandres and J. C. Gutiérrez-Vega, "Classical solutions for a free particle in a confocal elliptic billiard," American Journal of Physics 72, 810-817 (2004).

${ }^{6}$ Y. Sinai, "Doklady akademii nauk," (1963) pp. 1261-1264.

${ }^{7}$ L. A. Bunimovich, "Conditions of stochasticity of two-dimensional billiards," Chaos: An Interdisciplinary Journal of Nonlinear Science 1, 187193 (1991).

${ }^{8}$ A. Bäcker and H. R. Dullin, "Symbolic dynamics and periodic orbits for the cardioid billiard," Journal of Physics A: Mathematical and General 30, 1991-2020 (1997).

${ }^{9}$ L. A. Bunimovich, "Mushrooms and other billiards with divided phase space," Chaos: An Interdisciplinary Journal of Nonlinear Science 11, 802 808 (2001).

${ }^{10}$ A. Bäcker, R. Ketzmerick, S. Löck, M. Robnik, G. Vidmar, R. Höhmann, U. Kuhl, and H.-J. Stöckmann, "Dynamical tunneling in mushroom billiards," Physical Review Letters 100 (2008), 10.1103/physrevlett.100.174103.

${ }^{11}$ L. A. Bunimovich and L. V. Vela-Arevalo, "Some new surprises in chaos," Chaos: An Interdisciplinary Journal of Nonlinear Science 25, 097614 (2015).

${ }^{12}$ Y. G. Sinai, "Dynamical systems with elastic reflections," Russian Mathematical Surveys 25, 137-189 (1970).

${ }^{13}$ C. P. Dettmann, "Recent advances in open billiards with some open problems," in World Scientific Series on Nonlinear Science Series B (World Scientific, 2011) pp. 195-218.

${ }^{14}$ J. J. Lissauer, "Chaotic motion in the solar system," Reviews of Modern Physics 71, 835-845 (1999).

${ }^{15}$ J. Laskar, "Large scale chaos and marginal stability in the solar system," Celestial Mechanics and Dynamical Astronomy 64, 115-162 (1996).

${ }^{16} \mathrm{M}$. V. Berry, "Regular and irregular motion," in AIP Conference Proceedings (AIP, 1978).

${ }^{17}$ C. P. Dettmann, "Diffusion in the lorentz gas," Communications in Theoretical Physics 62, 521-540 (2014).

${ }^{18} \mathrm{G}$. D. Birkhoff, "On the periodic motions of dynamical systems," Acta Mathematica 50, 359-379 (1927).

${ }^{19}$ J. S. Portela, I. L. Caldas, and R. L. Viana, "Tokamak magnetic field lines described by simple maps," The European Physical Journal Special Topics 165, 195-210 (2008).

${ }^{20} \mathrm{~F}$. Haake, Quantum Signatures of Chaos (Springer Berlin Heidelberg, 2010).

${ }^{21}$ A. H. Barnett and T. Betcke, "Quantum mushroom billiards," Chaos: An Interdisciplinary Journal of Nonlinear Science 17, 043125 (2007).

${ }^{22}$ I. C. Percival, "Regular and irregular spectra," Journal of Physics B: Atomic and Molecular Physics 6, L229-L232 (1973).

${ }^{23}$ E. Persson, I. Rotter, H.-J. Stöckmann, and M. Barth, "Observation of resonance trapping in an open microwave cavity," Physical Review Letters 85, 2478-2481 (2000).

${ }^{24} \mathrm{~J}$. P. Bird, "Recent experimental studies of electron transport in open quantum dots," Journal of Physics: Condensed Matter 11, R413-R437 (1999).

${ }^{25}$ V. Milner, J. L. Hanssen, W. C. Campbell, and M. G. Raizen, "Optical billiards for atoms," Physical Review Letters 86, 1514-1517 (2001).

${ }^{26}$ N. Friedman, A. Kaplan, D. Carasso, and N. Davidson, "Observation of 
chaotic and regular dynamics in atom-optics billiards," Physical Review Letters 86, 1518-1521 (2001).

${ }^{27}$ B. Dietz, T. Friedrich, M. Miski-Oglu, A. Richter, and F. Schäfer, "Spectral properties of bunimovich mushroom billiards," Physical Review E 75 (2007), 10.1103/physreve.75.035203.

${ }^{28}$ C. Dembowski, H.-D. Gräf, A. Heine, R. Hofferbert, H. Rehfeld, and A. Richter, "First experimental evidence for chaos-assisted tunneling in a microwave annular billiard," Physical Review Letters 84, 867-870 (2000).

${ }^{29}$ R. P. Taylor, R. Newbury, A. S. Sachrajda, Y. Feng, P. T. Coleridge, C. P. Dettmann, N. Zhu, H.Guo, A. Delage, P. J. Kelly, and Z. Wasilewski, "Selfsimilar magnetoresistance of a semiconductor sinai billiard," Physical Review Letters 78, 1952-1955 (1997).

${ }^{30}$ R. Crook, C. G. Smith, A. C. Graham, I. Farrer, H. E. Beere, and D. A. Ritchie, "Imaging fractal conductance fluctuations and scarred wave functions in a quantum billiard," Physical Review Letters 91 (2003), 10.1103/physrevlett.91.246803.

${ }^{31}$ Č. Lozej and M. Robnik, "Structure, size, and statistical properties of chaotic components in a mixed-type hamiltonian system," Physical Review E 98 (2018), 10.1103/physreve.98.022220.

${ }^{32}$ C. F. Karney, "Long-time correlations in the stochastic regime," Physica D: Nonlinear Phenomena 8, 360-380 (1983).

${ }^{33}$ G. Cristadoro and R. Ketzmerick, "Universality of algebraic decays in hamiltonian systems," Physical Review Letters 100 (2008), 10.1103/physrevlett.100.184101.

${ }^{34}$ E. G. Altmann, A. E. Motter, and H. Kantz, "Stickiness in mushroom billiards," Chaos: An Interdisciplinary Journal of Nonlinear Science 15, 033105 (2005).

${ }^{35}$ L. A. Bunimovich and L. V. Vela-Arevalo, "Many faces of stickiness in hamiltonian systems," Chaos: An Interdisciplinary Journal of Nonlinear Science 22, 026103 (2012).

${ }^{36} \mathrm{C}$. Dettmann, "How sticky is the chaos/order boundary?" arXiv preprint arXiv:1603.00667 (2016)

${ }^{37}$ F. Vivaldi, G. Casati, and I. Guarneri, "Origin of long-time tails in strongly chaotic systems," Physical Review Letters 51, 727-730 (1983).

${ }^{38} \mathrm{G}$. Zaslavsky, "Chaos, fractional kinetics, and anomalous transport," Physics Reports 371, 461-580 (2002).

${ }^{39}$ C. V. Abud and R. E. de Carvalho, "Multifractality, stickiness, and recurrence-time statistics," Physical Review E 88 (2013), 10.1103/physreve.88.042922.

${ }^{40}$ Y. Zou, M. Thiel, M. C. Romano, and J. Kurths, "Characterization of stickiness by means of recurrence," Chaos: An Interdisciplinary Journal of Nonlinear Science 17, 043101 (2007).

${ }^{41} \mathrm{H}$. Poincaré, "Sur le problème des trois corps et les équations de la dynamique," Acta mathematica 13, 1-270 (1890).

${ }^{42} \mathrm{H}$. Furstenberg, "Poincaré recurrence and number theory," Bulletin of the American Mathematical Society 5, 211-235 (1981).

${ }^{43}$ N. Marwan, M. Carmenromano, M. Thiel, and J. Kurths, "Recurrence plots for the analysis of complex systems," Physics Reports 438, 237-329 (2007).

${ }^{44}$ J. Eckmann, S. O. Kamphorst, and D. Ruelle, "Recurrence plots of dynamical systems," Europhysics Letters (EPL) 4, 973-977 (1987).

${ }^{45}$ P. M. Addo, M. Billio, and D. Guégan, "Nonlinear dynamics and recurrence plots for detecting financial crisis," The North American Journal of Economics and Finance 26, 416-435 (2013).

${ }^{46}$ J. P. Zbilut, N. Thomasson, and C. L. Webber, "Recurrence quantification analysis as a tool for nonlinear exploration of nonstationary cardiac sig- nals," Medical Engineering \& Physics 24, 53-60 (2002).

${ }^{47} \mathrm{~L}$. Parrott, "Analysis of simulated long-term ecosystem dynamics using visual recurrence analysis," Ecological Complexity 1, 111-125 (2004).

${ }^{48} \mathrm{~N}$. Marwan and A. Meinke, "Extended recurrence plot analysis and its application to erp data," International Journal of Bifurcation and Chaos 14, 761-771 (2004).

${ }^{49}$ N. Asghari, C. Broeg, L. Carone, R. Casas-Miranda, J. C. C. Palacio, I. Csillik, R. Dvorak, F. Freistetter, G. Hadjivantsides, H. Hussmann, A. Khramova, M. Khristoforova, I. Khromova, I. Kitiashivilli, S. Kozlowski, T. Laakso, T. Laczkowski, D. Lytvinenko, O. Miloni, R. Morishima, A. Moro-Martin, V. Paksyutov, A. Pal, V. Patidar, B. Pečnik, O. Peles, J. Pyo, T. Quinn, A. Rodriguez, C. Romano, E. Saikia, J. Stadel, M. Thiel, N. Todorovic, D. Veras, E. V. Neto, J. Vilagi, W. von Bloh, R. Zechner, and E. Zhuchkova, "Stability of terrestrial planets in the habitable zone of gl 777 a, HD 72659, gl 614, 47 uma and HD 4208," Astronomy \& Astrophysics 426, 353-365 (2004).

${ }^{50} \mathrm{~S}$. Ranković and M. Porter, "Two-particle circular billiards versus randomly perturbed one-particle circular billiards," Chaos: An Interdisciplinary Journal of Nonlinear Science 23, 013123 (2013).

${ }^{51}$ R. S. Baroni, R. E. de Carvalho, B. Castaldi, and B. Furlanetto, "Time recurrence analysis of a near singular billiard," Mathematical and Computational Applications 24, 50 (2019).

${ }^{52}$ A. Rockett and P. Szüsz, Continued fractions (World Scientific Publishing Company, 1992).

${ }^{53}$ J. M. Greene, "A method for determining a stochastic transition," Journal of Mathematical Physics 20, 1183-1201 (1979).

${ }^{54}$ A. Paul and P. H. Richter, "Application of greenes method and the MacKay residue criterion to the double pendulum," Zeitschrift fur Physik B Condensed Matter 93, 515-520 (1994).

${ }^{55}$ M. Robnik, "Classical dynamics of a family of billiards with analytic boundaries," Journal of Physics A: Mathematical and General 16, 39713986 (1983).

${ }^{56}$ J. Szezech, S. Lopes, and R. Viana, "Finite-time lyapunov spectrum for chaotic orbits of non-integrable hamiltonian systems," Physics Letters A 335, 394-401 (2005).

${ }^{57} \mathrm{~N}$. Marwan, Encounters with neighbours: current developments of concepts based on recurrence plots and their applications (Norbert Marwan, 2003).

${ }^{58}$ Y. Zou, M. Thiel, M. C. Romano, and J. Kurths, "Analytical description of recurrence plots of dynamical systems with nontrivial recurrences," International Journal of Bifurcation and Chaos 17, 4273-4283 (2007).

${ }^{59}$ T. V. Ravenstein, "The three gap theorem (steinhaus conjecture)," Journal of the Australian Mathematical Society (Series A) 45, 360-370 (1988).

${ }^{60}$ C. P. Dettmann and O. Georgiou, "Open mushrooms: stickiness revisited," Journal of Physics A: Mathematical and Theoretical 44, 195102 (2011).

${ }^{61}$ M. Firmbach, S. Lange, R. Ketzmerick, and A. Bäcker, "Threedimensional billiards: Visualization of regular structures and trapping of chaotic trajectories," Physical Review E 98 (2018), 10.1103/physreve.98.022214.

${ }^{62}$ A. M. Fox and J. D. Meiss, "Greene's residue criterion for the breakup of invariant tori of volume-preserving maps," Physica D: Nonlinear Phenomena 243, 45-63 (2013).

${ }^{63} \mathrm{~J}$. Zbilut and C. W. Jr, "Embeddings and delays as derived from quantification of recurrence plots," Physics letters A 171, 199-203 (1992).

${ }^{64} \mathrm{~K}$. Falconer, Fractal geometry: mathematical foundations and applications (John Wiley \& Sons, 2004). 